\title{
Nonlinear Stability of the Triangular Libration Points for Radiating and Oblate Primaries in CR3BP in Nonresonance Condition
}

\author{
Nutan Singh ${ }^{1}$ and A. Narayan ${ }^{2}$ \\ ${ }^{1}$ Department of Mathematics, Rungta College of Engineering and Technology, Bhilai 490024, India \\ ${ }^{2}$ Department of Mathematics, Bhilai Institute of Technology, Durg 491001, India
}

Correspondence should be addressed to Nutan Singh; sendtonutan@gmail.com

Received 1 April 2015; Revised 17 June 2015; Accepted 21 July 2015

Academic Editor: Zdzislaw E. Musielak

Copyright (C) 2015 N. Singh and A. Narayan. This is an open access article distributed under the Creative Commons Attribution License, which permits unrestricted use, distribution, and reproduction in any medium, provided the original work is properly cited.

\begin{abstract}
This paper investigates the existence of resonance and nonlinear stability of the triangular equilibrium points when both oblate primaries are luminous. The study is carried out near the resonance frequency, satisfying the conditions $\omega_{1}=\omega_{2}, \omega_{1}=2 \omega_{2}$, and $\omega_{1}=3 \omega_{2}$ in circular cases by the application of Kolmogorov-Arnold-Moser (KAM) theory. The study is carried out for the various values of radiation pressure and oblateness parameters in general. It is noticed that the system experiences resonance at $\omega_{1}=2 \omega_{2}, \omega_{1}=3 \omega_{2}$ for different values of radiation pressures and oblateness parameter. The case $\omega_{1}=\omega_{2}$ corresponds to the boundary region of the stability for the system. It is found that, except for some values of the radiation pressure, and oblateness parameters and for $\mu \leq \mu_{c}=0.0385209$, the triangular equilibrium points are stable.
\end{abstract}

\section{Introduction}

The three-body problem, which describes three masses interacting through Newtonian gravity, has attracted many scientists for more than 300 years. The three-body problem is one of the most challenging problems in the history of science. In celestial mechanics the general three-body problem deals with gravitationally interacting astronomical bodies and intends to predict their motions. The restricted three-body problem (R3BP) is a particular case of the general three-body problem. The problem is restricted in the sense that the infinitesimal mass does not influence the motion of two gravitating primaries but is rather influenced by them. In R3BP, there are two possibilities; namely, the two bodies with dominant masses move around their common centre of mass along either circular or elliptic orbits, which lead to the respective circular or elliptic restricted threebody problems (ER3BP). The ER3BP describes the threedimensional motion of small particle called the infinitesimal mass under the gravitational force of two finite bodies called primaries around their common centre of mass. The
ER3BP generalizes the original circular restricted three-body problems, while some useful properties of circular model still can be satisfied in the elliptical case. The ER3BP describes the dynamical system more accurately as the primaries move along the elliptical orbit.

Modern applications of the three-body problem and restricted three-body problem have been extended to include the Earth, the Moon, and the artificial satellites as well as recently discovered exoplanets. The study of the stability of an elliptical or circular restricted three- body problem of the Hamiltonian system is generally performed by using Kolmogorov-Arnold-Moser (KAM) theorem. The KAM theory is very useful for studying the global stability in threebody problem. The KAM theorem is suitable for the motion undergoing small perturbations which preserves the features of the unperturbed motion.

The nonlinear stability for resonance as well as for the nonresonance cases of the triangular libration points, taking one of the bodies as radiating, was studied by Manju and Choudhry [1]. Kumar and Choudhry [2] investigated the stability of the triangular libration points for nonresonance 
as well as resonance case, taking both the bodies as radiating in circular restricted three-body problem in the presence of the third and fourth order resonance. Bhatnagar et al. [3] discussed the nonlinear stability of the triangular equilibrium points in circular restricted three bodies, considering bigger primary as a source of radiation. The nonlinear stability of the triangular Lagrangian points, considering the bigger primary as oblate spheroid in circular case, was examined by Markellos et al. [4]. Recently Narayan and Singh [5] studied the nonlinear stability of higher order for both radiating primaries and found that binary systems are stable.

The detailed description and the behavior of equilibrium points in ER3BP are touched upon by Danby [6], Bennett [7], Szebebely [8], Markeev [9], Selaru and Cucu-Dumitrescu [10], and Hallan and Rana [11]. The influence of the eccentricity of the orbits of the primaries with or without radiation pressure on the existence and stability of the equilibrium points was studied by Györgyey [12], Grebenikov [13], Kumar and Choudhry $[2,14]$, Markellos $[4,15]$, Sahoo and Ishwar [16], Roberts [17], Zimovshchikov and Tkhai [18], Ammar [19], Érdi et al. [20], Kumar and Ishwar [21], Singh and Umar $[22,23]$, Usha et al. [24], and Narayan and Singh [5, 25, 26].

It may be noted that the case when the frequencies are equal to zero or are equal to each other usually corresponds to the boundary of stability of the linear system, since, in the absence of oblateness parameters and radiation pressures the critical value of mass ratio denoted by $\mu_{c}$ is 0.0385209 . Also, if it is considered that $\omega_{1}>\omega_{2}>0$, then it is found that the inequality $n_{1} \omega_{1}+n_{2} \omega_{2} \neq 0$, provided $\left|n_{1}\right|+\left|n_{2}\right| \leq 4\left(n_{j}\right.$ are integers), is invalid for $\omega_{1}=2 \omega_{2}, \omega_{1}=3 \omega_{2}$ which gives rise to the resonance cases.

The present paper investigates the existence of the resonance and the stability of the infinitesimal mass about the triangular equilibrium points by taking both primaries as radiating and oblate in nonresonance condition. The existence of the resonance and the stability of infinitesimal near the resonance frequency satisfying the conditions $\omega_{1}=$ $\omega_{2}, \omega_{1}=2 \omega_{2}, \omega_{1}=3 \omega_{2}$ are studied in the circular cases. The study is carried out at various values of radiation pressures and oblateness parameters.

This paper has been organized in various sections.

Section 1 gives introduction, Section 2 describes the equations of motion of the problem, and Section 3 deals with the characteristics roots and first order stability of the triangular equilibrium points. The existence of resonance is discussed in Section 4, while Section 5 deals with normalization and higher order stability of the libration points. Finally Section 6 summarizes the discussion and conclusion of the paper.

\section{Equations of Motion}

The differential equations of the motion of the infinitesimal mass in elliptical restricted three-body problem under radiating primaries in pulsating system as given by Narayan and Shrivastava [27] are

$$
\begin{aligned}
& x^{\prime \prime}-2 y^{\prime}=\phi \Omega_{x} \\
& y^{\prime \prime}-2 x^{\prime}=\phi \Omega_{y},
\end{aligned}
$$

where the force function is defined as

$$
\begin{aligned}
\Omega & =\frac{x^{2}+y^{2}}{2}+\frac{1}{1+3\left(\left(A_{1}+A_{2}\right) / 2\right)}\left[\frac{(1-\mu) Q_{1}}{r_{1}}\right. \\
& \left.+\frac{\mu Q_{2}}{r_{2}}+\frac{(1-\mu) Q_{1} A_{1}}{2 r_{1}^{3}}+\frac{\mu A_{2} Q_{2}}{2 r_{2}^{3}}\right], \\
r_{1}^{2} & =(x+\mu)^{2}+y^{2} ; \\
r_{2}^{2} & =(x-1+\mu)^{2}+y^{2} ; \\
\phi & =\frac{1}{(1+e \cos f)},
\end{aligned}
$$

where $Q_{i}=1-\beta_{i}(i=1,2)$ is the radiation pressure, $f$ is a true anomaly of the primaries, $A_{1}, A_{2}$ are the oblateness parameter, $e$ is the eccentricity of the orbits, and $\mu$ is the mass ratio defined as

$$
\begin{gathered}
\mu=\frac{m_{2}}{m_{1}+m_{2}} ; \\
1-\mu=\frac{m_{1}}{m_{1}+m_{2}} ; \\
0 \leq \mu \leq \frac{1}{2} .
\end{gathered}
$$

The coordinates of the triangular equilibrium points $L_{4}$ and $L_{5}$ as given by Narayan and Shrivastava [27] are

$$
\begin{aligned}
x_{0} & =\frac{1}{2}-\mu+\frac{A_{1}}{2}-\frac{A_{2}}{2}+\frac{\beta_{2}}{3}-\frac{\beta_{1}}{3}-\frac{1}{2} A_{1} \beta_{1}+\frac{1}{2} \\
& \cdot A_{2} \beta_{2} ; \\
y_{o} & = \pm \frac{\sqrt{3}}{2}\left[1-\frac{A_{1}}{3}-\frac{A_{2}}{3}-\frac{2 \beta_{1}}{9}-\frac{2 \beta_{2}}{9}-\frac{1}{3} A_{1} \beta_{1}\right. \\
& \left.-\frac{1}{3} A_{2} \beta_{2}\right] .
\end{aligned}
$$

\section{Characteristics Roots and First Order Stability of the Triangular Equilibrium Points}

The stability of the elliptical restricted three-body problem is restricted to planar case only. Since the nature of stability about $L_{4}$ and $L_{5}$ is similar it is sufficient to study the stability only about $L_{4}$. The Hamiltonian as described by Narayan and Shrivastava [27] is given by

$$
\begin{aligned}
H & =\frac{p_{1}^{2}+p_{2}^{2}}{2}+\left(p_{1} q_{2}-p_{2} q_{1}\right)+\frac{\left(q_{1}^{2}+q_{2}^{2}\right) e \cos f}{2(1+e \cos f)} \\
& -\frac{1}{(1+e \cos f)\left(1+3\left(A_{1}+A_{2}\right) / 2\right)}\left\{\left\{\frac{(1-\mu) Q_{1}}{r_{1}}\right.\right. \\
& \left.\left.+\frac{\mu Q_{2}}{r_{2}}+\frac{(1-\mu) Q_{1} A_{1}}{2 r_{1}^{3}}+\frac{\mu A_{2} Q_{2}}{2 r_{2}^{3}}\right\}\right\},
\end{aligned}
$$

where $p_{1}, p_{2}, q_{1}, q_{2}$ are the variations in the coordinates $L_{4}$. 
By substituting $Q_{i}=1-\beta_{i}(i=1,2)$ we have

$$
\begin{aligned}
H & =\frac{p_{1}^{2}+p_{2}^{2}}{2}+\left(p_{1} q_{2}-p_{2} q_{1}\right)+\frac{\left(q_{1}^{2}+q_{2}^{2}\right) e \cos f}{2(1+e \cos f)} \\
& -\frac{1}{(1+e \cos f)\left(1+3\left(A_{1}+A_{2}\right) / 2\right)}\left\{\frac{(1-\mu)\left(1-\beta_{1}\right)}{r_{1}}\right. \\
& \left.+\frac{\mu\left(1-\beta_{2}\right)}{r_{2}}+\frac{(1-\mu)\left(1-\beta_{1}\right) A_{1}}{2 r_{1}^{3}}+\frac{\mu A_{2}\left(1-\beta_{2}\right)}{2 r_{2}^{3}}\right\} .
\end{aligned}
$$

Now expand the Hamiltonian function as

$$
H=\sum_{K=0}^{\infty} H_{K}=H_{0}+H_{1}+H_{2}+H_{3}+\cdots+H_{4}+\cdots,
$$

where $H_{K}$ is the sum of the terms of the $K$ th degree which is homogeneous in the variables $p_{1}, p_{2}, q_{1}, q_{2} . H_{0}$ is constant and $H_{1}=0$. Consider

$$
\begin{aligned}
H_{2} & \\
= & \frac{p_{1}^{2}+p_{2}^{2}}{2}+\left(p_{1} q_{2}-p_{2} q_{1}\right) \\
& +\frac{e \cos f}{2(1+e \cos f)\left\{1+3\left(\left(A_{1}+A_{2}\right) / 2\right)\right\}}\left(q_{1}^{2}+q_{2}^{2}\right) \\
& -\frac{1}{2(1+e \cos f)}\left(H_{20} q_{1}^{2}-H_{11} q_{1} q_{2}-H_{02} q_{2}^{2}\right),
\end{aligned}
$$

where

$$
\begin{aligned}
H_{20} & =-\left(\frac{-1}{8}+\frac{9}{8} A_{1}-\frac{9}{16} A_{2}-\frac{73}{24} A_{1} \beta_{1}+\frac{11}{48} A_{2} \beta_{2}\right. \\
& -\frac{27}{16} \mu A_{1}+\frac{27}{16} \mu A_{2}+\frac{17}{8} \mu \beta_{1}-\frac{7}{9} \mu \beta_{2}+\frac{\beta_{2}}{2} \\
& \left.-\frac{13 \beta_{1}}{8}\right), \\
H_{11} & =\sqrt{3}\left(\frac{3}{4}+\frac{17}{4} A_{1}+\frac{7}{8} A_{2}+\frac{139}{24} A_{1} \beta_{1}+\frac{15}{8} A_{2} \beta_{2}\right. \\
- & \frac{41}{8} \mu A_{1}-\frac{41}{8} \mu A_{2}-\frac{1}{6} \mu \beta_{1}+\mu \beta_{2}-\frac{3 \mu}{2}-\frac{\beta_{2}}{3} \\
- & \left.\frac{7 \beta_{1}}{12}\right), \\
H_{02} & =\left(\frac{5}{8}+\frac{27}{8} A_{1}+\frac{21}{16} A_{2}+\frac{107}{16} A_{1} \beta_{1}+\frac{11}{16} A_{2} \beta_{2}\right. \\
& -\frac{33}{8} \mu A_{1}+\frac{33}{16} \mu A_{2}-\frac{7}{8} \mu \beta_{1}+\frac{11}{8} \mu \beta_{2}-\frac{\beta_{2}}{2} \\
+ & \left.\frac{7 \beta_{1}}{8}\right),
\end{aligned}
$$

$$
\begin{aligned}
& H_{3}=\frac{-1}{6(1+e \cos f)\left[1+3\left(A_{1}+A_{2}\right) / 2\right]}\left[q _ { 1 } ^ { 3 } \left(\frac{21}{8}\right.\right. \\
& +\frac{15 A_{1}}{4}-\frac{295}{16} A_{2}-\frac{93 \beta_{1}}{4}-\frac{65 \beta_{2}}{6}-\frac{365 \beta_{1} A_{1}}{24} \\
& -\frac{155 \beta_{1} A_{2}}{16}+\mu\left(-\frac{57}{8}-\frac{15 A_{1}}{4}-\frac{305 A_{2}}{16}-\frac{29 \beta_{1}}{4}\right. \\
& +\frac{51 \beta_{2}}{8}-\frac{365 \beta_{1} A_{1}}{24}+\frac{85 \beta_{1} A_{2}}{16}-\frac{17 \beta_{2} A_{1}}{8} \\
& \left.\left.-\frac{5 \beta_{2} A_{2}}{3}\right)\right)+3 q_{1}^{2} q_{2}\left(\frac{-3}{8}+\frac{21 A_{1}}{8}-\frac{61}{8} A_{2}\right. \\
& -\frac{113 \beta_{1}}{24}+\frac{7 \beta_{2}}{4}-\frac{53 \beta_{1} A_{1}}{24}+\frac{61 \beta_{1} A_{2}}{8} \\
& +\mu \sqrt{3}\left(\frac{17 A_{1}}{16}+\frac{9 A_{2}}{16}+\frac{47 \beta_{1}}{6}-\frac{13 \beta_{2}}{4}-\frac{53 \beta_{1} A_{1}}{24}\right. \\
& \left.\left.-\frac{113 \beta_{1} A_{2}}{24}-\frac{91 \beta_{2} A_{1}}{16}-\frac{\beta_{2} A_{2}}{48}\right)\right)+3 q_{1} q_{2}^{2}\left(\frac{-33}{8}\right. \\
& -\frac{315 A_{1}}{8}+\frac{5}{12} A_{2}+\frac{149 \beta_{1}}{24}+\frac{5 \beta_{2}}{8}-\frac{265 \beta_{1} A_{1}}{8} \\
& -\frac{5 \beta_{1} A_{2}}{12}+\mu\left(\frac{33}{4}-\frac{245 A_{1}}{8}+\frac{263 A_{2}}{8}+\frac{77 \beta_{1}}{48}\right. \\
& -\frac{311 \beta_{2}}{16}-\frac{55 \beta_{1} A_{1}}{4}+\frac{3805 \beta_{1} A_{2}}{96}+\frac{55 \beta_{2} A_{1}}{8} \\
& \left.\left.-\frac{1441 \beta_{2} A_{2}}{16}\right)\right)+q_{2}^{3}\left(\frac{-9}{8}-\frac{51 A_{1}}{4}-\frac{69}{16} A_{2}\right. \\
& -\frac{13 \beta_{1}}{8}-\frac{11 \beta_{2}}{4}+\frac{151 \beta_{1} A_{1}}{16}+\frac{65 \beta_{1} A_{2}}{16} \\
& +\mu\left(\frac{195 A_{1}}{16}-\frac{33 A_{2}}{2}+\frac{81 \beta_{1}}{16}+\frac{31 \beta_{2}}{8}-\frac{151 \beta_{1} A_{1}}{16}\right. \\
& \left.\left.\left.+\frac{117 \beta_{1} A_{2}}{32}-\frac{317 \beta_{2} A_{1}}{32}+\frac{5 \beta_{2} A_{2}}{4}\right)\right)\right], \\
& H_{4}=\frac{-1}{24(1+e \cos f)}\left[1+\frac{3\left(A_{1}+A_{2}\right)}{2}\right]\left[q _ { 1 } ^ { 4 } \left(\frac{-111}{16}\right.\right. \\
& -\frac{1155 A_{1}}{16}+\frac{9815}{16} A_{2}+\frac{397 \beta_{1}}{2}-\frac{25 \beta_{2}}{2} \\
& -\frac{1235 \beta_{1} A_{1}}{16}-\frac{1185 \beta_{1} A_{2}}{2}+\mu\left(-\frac{1575}{16}-\frac{395 A_{1}}{48}\right. \\
& -\frac{29545 A_{2}}{48}+\frac{9269 \beta_{1}}{16}+\frac{439 \beta_{2}}{144}-\frac{1234 \beta_{1} A_{1}}{16} \\
& \left.\left.-\frac{6325 \beta_{1} A_{2}}{12}+\frac{6755 \beta_{2} A_{1}}{48}+\frac{605 \beta_{2} A_{2}}{48}\right)\right) \\
& +4 \sqrt{3} q_{1}^{3} q_{2}\left(\frac{-75}{16}-\frac{281 A_{1}}{16}-\frac{21}{2} A_{2}+\frac{5 \beta_{1}}{16}\right. \\
& +\frac{21 \beta_{2}}{6}+\frac{351 \beta_{1} A_{1}}{16}+\frac{21 \beta_{1} A_{2}}{2}+\mu\left(\frac{75}{8}\right.
\end{aligned}
$$




$$
\begin{aligned}
& +\frac{5669 A_{1}}{208}-\frac{117 A_{2}}{8}-\frac{235 \beta_{1}}{48}-\frac{6121 \beta_{2}}{560}-\frac{7 \beta_{1} A_{1}}{2} \\
& \left.\left.+\frac{319 \beta_{1} A_{2}}{32}+\frac{2357 \beta_{2} A_{1}}{156}-\frac{83 \beta_{2} A_{2}}{4}\right)\right) \\
& +6 q_{1}^{2} q_{2}^{2}\left(\frac{123}{16}+\frac{4865 A_{1}}{48}-\frac{105}{8} A_{2}+\frac{235 \beta_{1}}{12}\right. \\
& +\frac{75 \beta_{2}}{12}-\frac{4295 \beta_{1} A_{1}}{48}+\frac{105 \beta_{1} A_{2}}{8}+\mu\left(-\frac{123}{8}\right. \\
& +\frac{1045 \beta_{1} A_{1}}{8}-\frac{14765 \beta_{1} A_{2}}{96}-\frac{1325 \beta_{2} A_{1}}{32} \\
& +\frac{5735 A_{1}}{48}+\frac{1601 A_{2}}{16}-\frac{421 \beta_{1}}{48}-\frac{31 \beta_{2}}{8} \\
& \left.\left.+\frac{175 \beta_{2} A_{2}}{48}\right)\right)+q_{2}^{4}\left(\frac{9}{16}+\frac{1125 A_{1}}{16}-\frac{1575}{32} A_{2}\right. \\
& +\frac{4865 \beta_{1} A_{1}}{48}+\frac{245 \beta_{1} A_{2}}{32}+\frac{45 \beta_{2} A_{1}}{8} \\
& +\frac{63 \beta_{2}}{16}-\frac{21 \beta_{2}}{5}+\frac{23 \beta_{1} A_{1}}{2}-\frac{15 \beta_{1} A_{2}}{4} \\
& +
\end{aligned}
$$

Restricting to $\mathrm{H}_{2}$ alone, the characteristics equation can be given in the form

$$
\left|\begin{array}{cc}
\lambda^{2}-\left(1-2 H_{20}\right) & -2 \lambda-H_{11} \\
2 \lambda-H_{11} & \lambda^{2}-\left(1+2 H_{02}\right)
\end{array}\right|=0,
$$

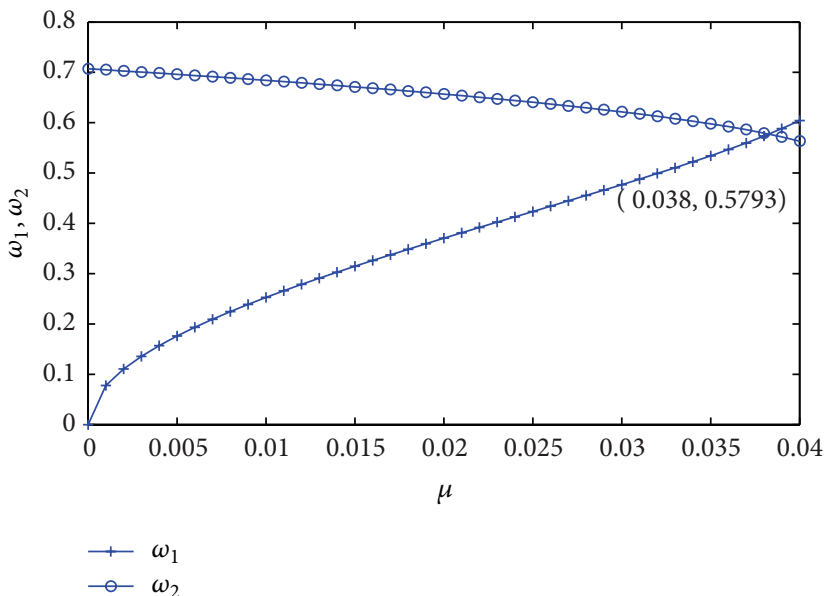

FIGURE 1: Correlation between $\mu$ and $\omega_{1}$ and $\omega_{2}$ with $\beta_{1}=0.01, \beta_{2}=$ $0.02, A_{1}=0.01$, and $A_{2}=0.02$.

where $H_{20}, H_{11}$, and $H_{02}$ are given by ((9), (10), and (11)). After further calculations the characteristics equation reduces to the form

$$
\begin{aligned}
& \lambda^{4}+\lambda^{2}+\left\{\frac{27 \mu(1-\mu)}{4}\right\} \\
& \cdot\left\{1-\frac{125}{6} A_{1}+\frac{40}{3} A_{2}+\frac{5}{6} \beta_{1}-\frac{35}{18} \beta_{2}\right\}=0 .
\end{aligned}
$$

If $\omega_{1}$ and $\omega_{2}$ are the frequencies then putting $\lambda^{2}=-\omega^{2}$ in (15), roots can be written as

$$
\begin{aligned}
& \omega_{1}^{2}=-\lambda_{1,2}^{2}=\frac{1}{2}[1+\{1-27 \mu(1-\mu) \\
& \left.\left.\cdot\left(1-\frac{125}{6} A_{1}+\frac{40}{3} A_{2}+\frac{5}{6} \beta_{1}-\frac{35}{18} \beta_{2}\right)\right\}^{1 / 2}\right], \\
& \omega_{2}^{2}=-\lambda_{3,4}^{2}=\frac{1}{2}[1-\{1-27 \mu(1-\mu) \\
& \left.\left.\cdot\left(1-\frac{125}{6} A_{1}+\frac{40}{3} A_{2}+\frac{5}{6} \beta_{1}-\frac{35}{18} \beta_{2}\right)\right\}^{1 / 2}\right] .
\end{aligned}
$$

The correlation between $\mu$ and $\omega_{1}$ and $\omega_{2}$ is shown in Figures $1-5$. It is found that for fixed values of radiation pressure $\omega_{1}$ increases with an increase in $\mu$ whereas $\omega_{2}$ decreases.

\section{Existence of Resonance in Circular Cases}

In order to discuss the existence of resonance, we consider the following three cases. 
Case 1. The first case is when $\omega_{1}=\omega_{2}$; that is,

$$
\lambda_{1,2}^{2}=\lambda_{3,4}^{2}
$$

Solving the above equation we obtain

$$
\begin{aligned}
1- & 27 \mu(1-\mu)\left(1-\frac{125}{6} A_{1}+\frac{40}{3} A_{2}+\frac{5}{6} \beta_{1}-\frac{35}{18} \beta_{2}\right) \\
=0 &
\end{aligned}
$$

that is,

$$
\begin{aligned}
27(1 & \left.-\frac{125}{6} A_{1}+\frac{40}{3} A_{2}+\frac{5}{6} \beta_{1}-\frac{35}{18} \beta_{2}\right) \mu^{2} \\
& -27\left(1-\frac{125}{6} A_{1}+\frac{40}{3} A_{2}+\frac{5}{6} \beta_{1}-\frac{35}{18} \beta_{2}\right) \mu \\
& +1=0 .
\end{aligned}
$$

Solving for $\mu$ we obtain

$$
\mu=\frac{9 \pm \sqrt{69}\left(1-(125 / 69) A_{1}+(80 / 69) A_{2}+(5 / 69) \beta_{1}-(35 / 207) \beta_{2}\right)}{18}
$$

Since $\mu \leq 1 / 2$, the positive sign is inadmissible. Hence the region of stability in the first approximation can be written as

$$
0<\mu<\frac{\left\{9-\sqrt{69}\left(1-(125 / 69) A_{1}+(80 / 69) A_{2}+(5 / 69) \beta_{1}-(35 / 207) \beta_{2}\right)\right\}}{18} .
$$

Thus, the value of $\mu$ responsible for stable equilibrium points is given by

$$
\begin{aligned}
\mu_{c}= & 0.0385208965+0.83601 A_{1}+0.535048 A_{2} \\
& -0.0334405 \beta_{1}+0.0780279 \beta_{2} .
\end{aligned}
$$

It is clear from (22) that in the absence of radiation pressures and oblateness parameters the critical value of mass ratio is 0.0385208965 when $\omega_{1}=\omega_{2}$, which usually corresponds to a boundary of the region of stability of the system.

Case 2. It is when $\omega_{1}=2 \omega_{2}$; that is,

$$
\lambda_{1,2}^{2}=4 \lambda_{3,4}^{2}
$$

that is,

$$
\begin{aligned}
& {[1+\{1-27 \mu(1-\mu)} \\
& \left.\left.\quad \cdot\left(1-\frac{125}{6} A_{1}+\frac{40}{3} A_{2}+\frac{5}{6} \beta_{1}-\frac{35}{18} \beta_{2}\right)\right\}^{1 / 2}\right]
\end{aligned}
$$

$$
\begin{aligned}
& =4[1-\{1-27 \mu(1-\mu) \\
& \left.\left.\cdot\left(1-\frac{125}{6} A_{1}+\frac{40}{3} A_{2}+\frac{5}{6} \beta_{1}-\frac{35}{18} \beta_{2}\right)\right\}^{1 / 2}\right] .
\end{aligned}
$$

Solving the above equation for $\mu$ the resonance value is obtained as

$$
\begin{aligned}
\mu_{02} & =\frac{1}{2} \\
& \pm \sqrt{\frac{1}{4}-\frac{16}{675\left(1-125 A_{1} / 6+40 A_{2} / 3+5 \beta_{1} / 6-35 \beta_{2} / 18\right)}} .
\end{aligned}
$$

Case 3. It is when $\omega_{1}=3 \omega_{2}$; that is,

$$
\lambda_{1,2}^{2}=9 \lambda_{3,4}^{2}
$$

that is,

$$
[1+\{1-27 \mu(1-\mu)
$$

$$
\begin{aligned}
& \left.\left.\cdot\left(1-\frac{125}{6} A_{1}+\frac{40}{3} A_{2}+\frac{5}{6} \beta_{1}-\frac{35}{18} \beta_{2}\right)\right\}^{1 / 2}\right] \\
& =9[1-\{1-27 \mu(1-\mu) \\
& \left.\left.\cdot\left(1-\frac{125}{6} A_{1}+\frac{40}{3} A_{2}+\frac{5}{6} \beta_{1}-\frac{35}{18} \beta_{2}\right)\right\}^{1 / 2}\right] .
\end{aligned}
$$




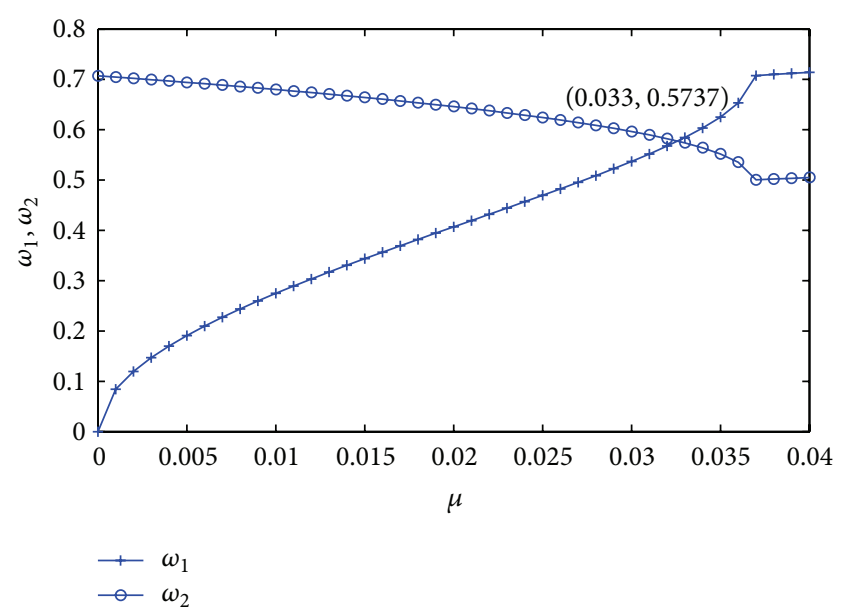

Figure 2: Correlation between $\mu$ and $\omega_{1}$ and $\omega_{2}$ with $\beta_{1}=0.01$, $\beta_{2}=0.02, A_{1}=0.03$, and $A_{2}=0.04$.

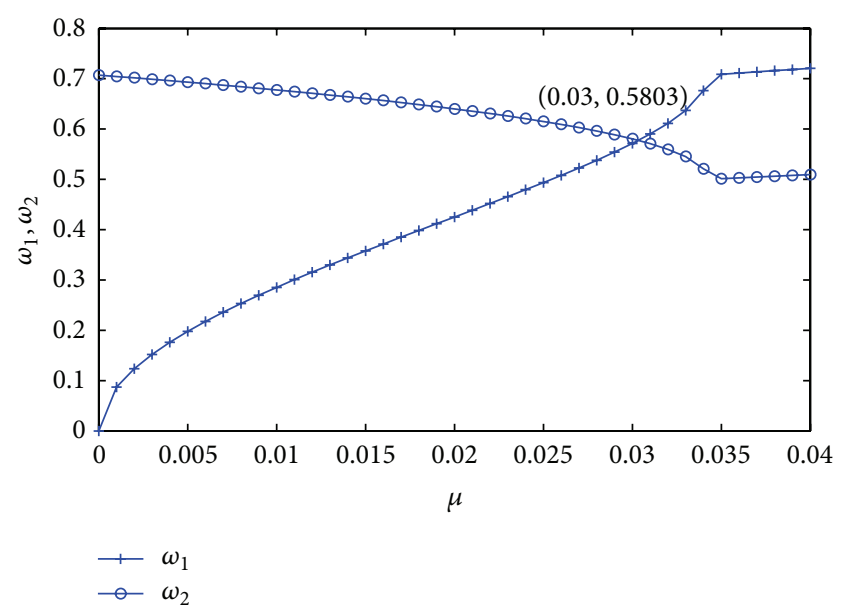

FIGURE 3: Correlation between $\mu$ and $\omega_{1}$ and $\omega_{2}$ with $\beta_{1}=0.01$, $\beta_{2}=0.02, A_{1}=0.04$, and $A_{2}=0.05$.

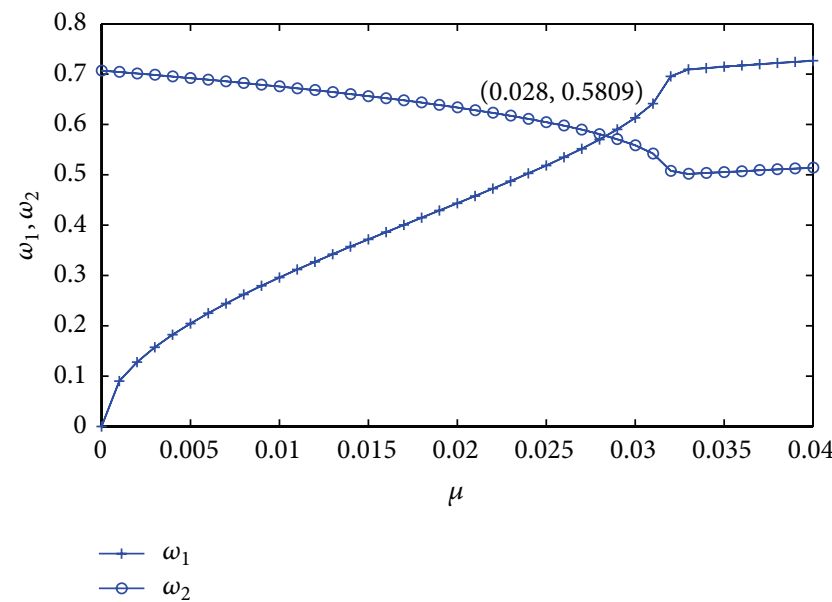

FIgURE 4: Correlation between $\mu$ and $\omega_{1}$ and $\omega_{2}$ with $\beta_{1}=0.01$, $\beta_{2}=0.02, A_{1}=0.05$, and $A_{2}=0.06$.

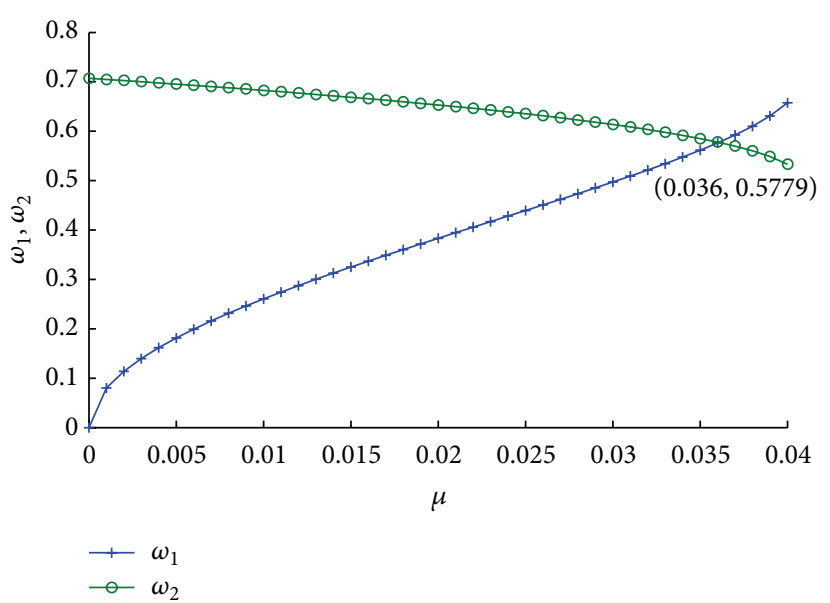

FIGURE 5: Correlation between $\mu$ and $\omega_{1}$ and $\omega_{2}$ with $\beta_{1}=0.01$, $\beta_{2}=0.02, A_{1}=0.001$, and $A_{2}=0.002$.

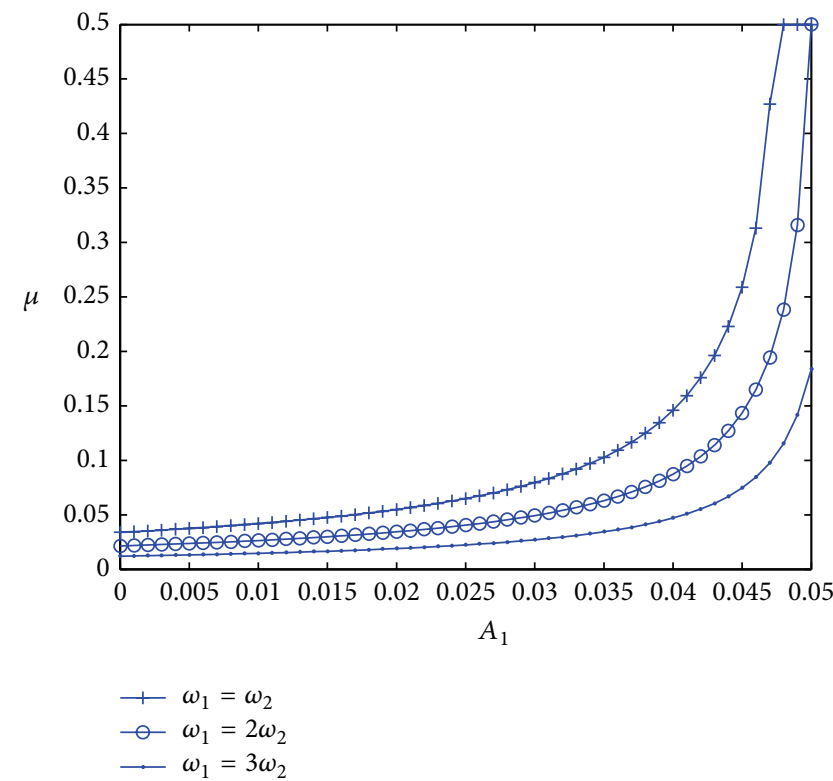

FIgURE 6: Correlation between $\mu$ and $A_{1}$ and $\beta_{1}=0.01, \beta_{2}=0.02$, and $A_{2}=0.01$.

Solving the above equation for $\mu$ the resonance value is obtained as

$$
\begin{aligned}
\mu_{03} & =\frac{1}{2} \\
& \pm \sqrt{\frac{1}{4}-\frac{9}{675\left(1-125 A_{1} / 6+40 A_{2} / 3+5 \beta_{1} / 6-35 \beta_{2} / 18\right)}} .
\end{aligned}
$$

Table 1 shows the values of $\mu$ corresponding to $\omega_{1}=\omega_{2}, \omega_{1}=$ $2 \omega_{2}$, and $\omega_{1}=3 \omega_{2}$. Figures 6-10 show correlation between $\mu$ and $A_{1}$ by varying $\beta_{1}, \beta_{2}$, and $A_{2}$. Correlation between $\mu$ and $\beta_{1}$ is depicted in Figure 11 by varying $\beta_{2}$ and taking $A_{1}=0.01$ and $A_{2}=0.02$. Figure 12 shows combined figures between $\mu$ and $\beta_{1}$ by taking $\beta_{2}=0.01, A_{1}=0.01$, and $A_{2}=0.01,0.02$, 0.03 . 
TABLE 1: Values of $\mu_{01}, \mu_{02}$, and $\mu_{03}$ for different values of radiation pressure and oblateness parameter.

\begin{tabular}{|c|c|c|c|c|c|c|}
\hline$\beta_{1}$ & $\beta_{2}$ & $A_{1}$ & $A_{2}$ & $\mu_{01}$ & $\mu_{02}$ & $\mu_{03}$ \\
\hline 0.01 & 0.01 & 0.01 & 0.02 & 0.0367 & 0.032 & 0.0129 \\
\hline 0.01 & 0.01 & 0.02 & 0.03 & 0.0397 & 0.0250 & 0.0139 \\
\hline 0.01 & 0.01 & 0.04 & 0.05 & 0.0473 & 0.0297 & 0.0165 \\
\hline 0.01 & 0.02 & 0.01 & 0.02 & 0.0374 & 0.0236 & 0.0131 \\
\hline 0.01 & 0.02 & 0.02 & 0.03 & 0.0405 & 0.0255 & 0.0142 \\
\hline 0.01 & 0.02 & 0.04 & 0.05 & 0.0485 & 0.0305 & 0.0169 \\
\hline 0.01 & 0.03 & 0.01 & 0.02 & 0.0367 & 0.0232 & 0.0129 \\
\hline 0.01 & 0.03 & 0.02 & 0.03 & 0.0441 & 0.0278 & 0.0154 \\
\hline 0.01 & 0.03 & 0.04 & 0.05 & 0.0498 & 0.0312 & 0.173 \\
\hline 0.01 & 0.04 & 0.01 & 0.02 & 0.0390 & 0.0246 & 0.0137 \\
\hline 0.01 & 0.04 & 0.02 & 0.03 & 0.0423 & 0.0266 & 0.0148 \\
\hline 0.01 & 0.04 & 0.04 & 0.05 & 0.0511 & 0.0321 & 0.0178 \\
\hline 0.01 & 0.05 & 0.01 & 0.02 & 0.0398 & 0.0251 & 0.0139 \\
\hline 0.01 & 0.05 & 0.02 & 0.03 & 0.0433 & 0.0272 & 0.0151 \\
\hline 0.01 & 0.05 & 0.04 & 0.05 & 0.0525 & 0.0329 & 0.0182 \\
\hline 0.01 & 0.06 & 0.01 & 0.02 & 0.0406 & 0.0256 & 0.0142 \\
\hline 0.01 & 0.06 & 0.02 & 0.03 & 0.0443 & 0.0279 & 0.0155 \\
\hline 0.01 & 0.06 & 0.04 & 0.05 & 0.0540 & 0.0338 & 0.0187 \\
\hline 0.01 & 0.07 & 0.01 & 0.02 & 0.0415 & 0.0262 & 0.0145 \\
\hline 0.01 & 0.07 & 0.02 & 0.03 & 0.0453 & 0.0285 & 0.0158 \\
\hline 0.01 & 0.07 & 0.04 & 0.05 & 0.0556 & 0.0348 & 0.0193 \\
\hline 0.01 & 0.08 & 0.01 & 0.02 & 0.0425 & 0.0267 & 0.0149 \\
\hline 0.01 & 0.08 & 0.02 & 0.03 & 0.0465 & 0.0292 & 0.0162 \\
\hline 0.01 & 0.08 & 0.04 & 0.05 & 0.0573 & 0.0358 & 0.0198 \\
\hline 0.01 & 0.09 & 0.01 & 0.02 & 0.0434 & 0.0273 & 0.0152 \\
\hline 0.01 & 0.09 & 0.02 & 0.03 & 0.0476 & 0.0299 & 0.0166 \\
\hline 0.01 & 0.09 & 0.04 & 0.05 & 0.0590 & 0.0369 & 0.0204 \\
\hline 0.87 & 0.79 & 0.01 & 0.02 & 0.1835 & 0.1074 & 0.0572 \\
\hline 0.87 & 0.79 & 0.02 & 0.03 & 0.3131 & 0.1648 & 0.0846 \\
\hline 0.87 & 0.79 & 0.04 & 0.05 & $0.5-1.1902 i$ & $0.5-0.9037 i$ & $0.5-0.5916 i$ \\
\hline 0.72 & 0.29 & 0.01 & 0.02 & 0.0351 & 0.0221 & 0.0123 \\
\hline 0.72 & 0.29 & 0.02 & 0.03 & 0.0378 & 0.0238 & 0.0133 \\
\hline 0.72 & 0.29 & 0.04 & 0.05 & 0.0409 & 0.0258 & 0.0143 \\
\hline 0.62 & 0.39 & 0.01 & 0.02 & 0.0476 & 0.0299 & 0.0166 \\
\hline 0.62 & 0.39 & 0.02 & 0.03 & 0.0527 & 0.0331 & 0.0183 \\
\hline 0.62 & 0.39 & 0.04 & 0.05 & 0.0671 & 0.0418 & 0.231 \\
\hline 0.52 & 0.49 & 0.01 & 0.02 & 0.0742 & 0.0461 & 0.0254 \\
\hline 0.52 & 0.49 & 0.02 & 0.03 & 0.0875 & 0.0540 & 0.0296 \\
\hline 0.52 & 0.49 & 0.04 & 0.05 & 0.1367 & 0.0823 & 0.0445 \\
\hline 0.82 & 0.29 & 0.01 & 0.02 & 0.0325 & 0.0205 & 0.0115 \\
\hline 0.82 & 0.29 & 0.02 & 0.03 & 0.0348 & 0.0220 & 0.0122 \\
\hline 0.82 & 0.29 & 0.04 & 0.05 & 0.0405 & 0.0255 & 0.0142 \\
\hline 0.92 & 0.29 & 0.01 & 0.02 & 0.0303 & 0.0192 & 0.0107 \\
\hline 0.92 & 0.29 & 0.02 & 0.03 & 0.0323 & 0.0204 & 0.0114 \\
\hline 0.92 & 0.29 & 0.04 & 0.05 & 0.0371 & 0.0234 & 0.0130 \\
\hline
\end{tabular}

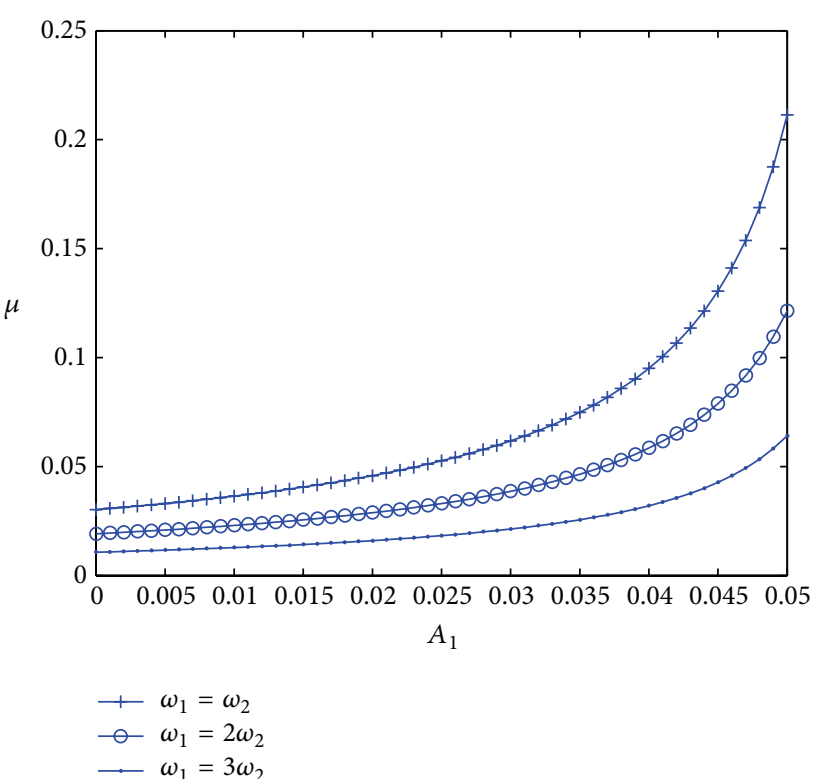

Figure 7: Correlation between $\mu$ and $A_{1}$ and $\beta_{1}=0.01, \beta_{2}=0.02$, and $A_{2}=0.02$.

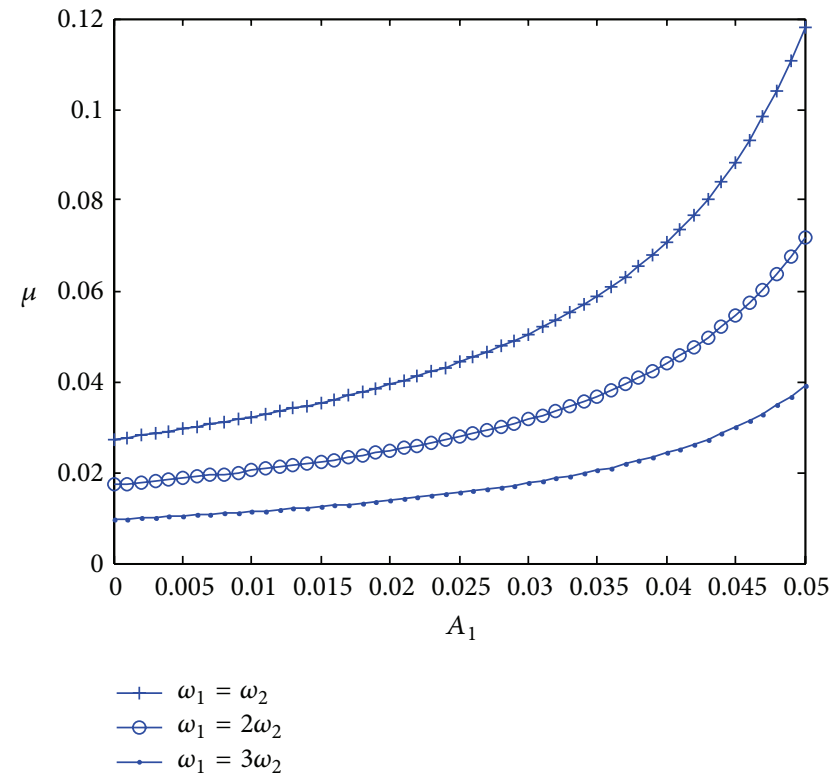

FIGURE 8: Correlation between $\mu$ and $A_{1}$ and $\beta_{1}=0.01, \beta_{2}=0.02$, and $A_{2}=0.03$.

\section{Normalization and Higher Order Stability of the Libration Points}

In order to investigate the stability the Hamiltonian $H$ is normalized by Birkhoff's method to the following form:

$$
\begin{aligned}
H= & \omega_{1} t_{1}-\omega_{2} t_{2}+c_{20} t_{1}^{2}+c_{11} t_{1} t_{2}+c_{02} t_{2}^{2} \\
& +o\left(t_{1}+t_{2}\right)^{5 / 2},
\end{aligned}
$$




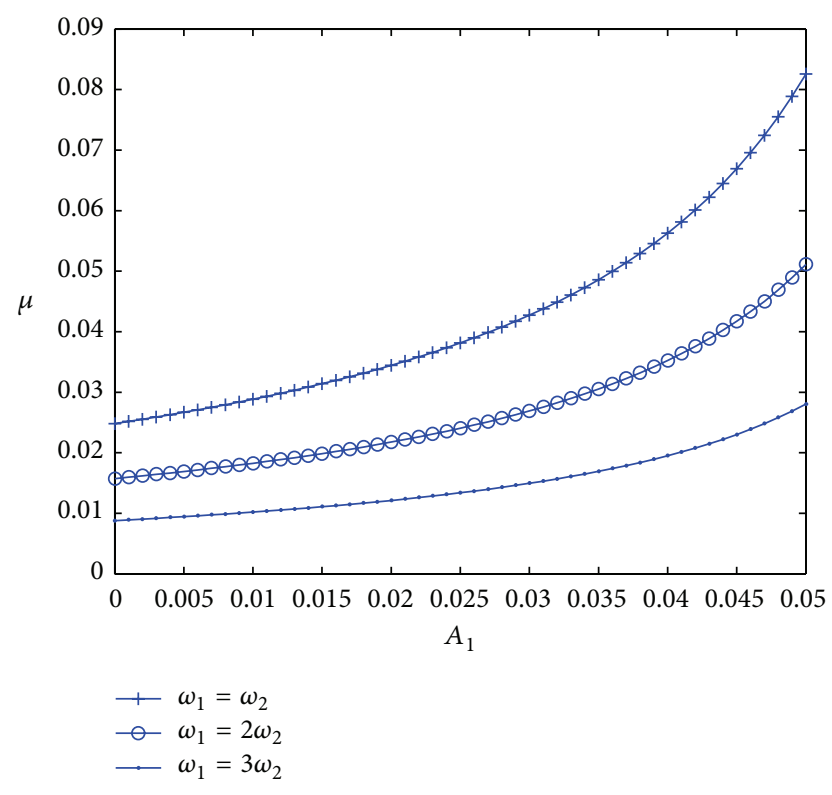

FIgURE 9: Correlation between $\mu$ and $A_{1}$ and $\beta_{1}=0.01, \beta_{2}=0.02$, and $A_{2}=0.04$.

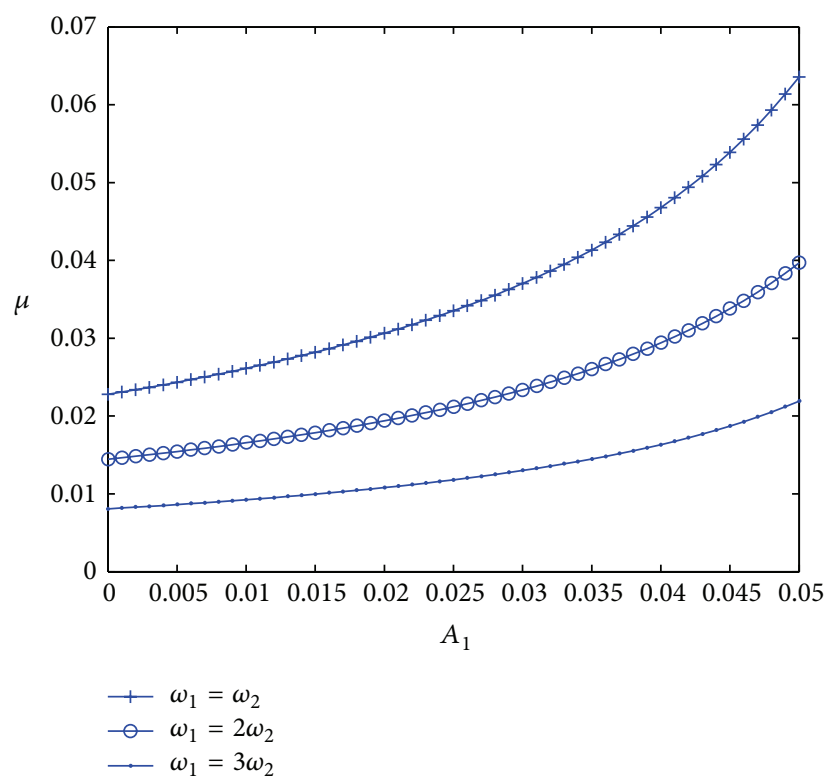

Figure 10: Correlation between $\mu$ and $A_{1}$ and $\beta_{1}=0.01, \beta_{2}=0.02$, and $A_{2}=0.05$.

where

$$
2 t_{i}=p_{i}^{2}+q_{i}^{2}, \quad i=1,2
$$

If $\mathrm{H}_{2}$ is Hamiltonian of 2 nd order, defined by (8), (9), (10), and (11) and is of a positive definite form, then the equilibrium position is stable by virtue of Lyapunov theorem [28]. Otherwise the problem of stability can be solved by KAM theorem as given by Arnold $[29,30]$. To apply KAM

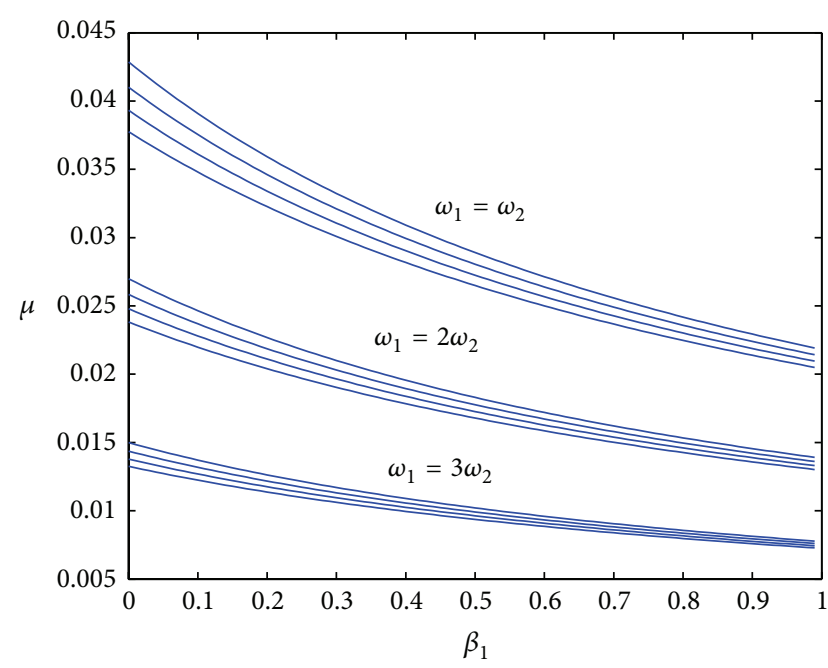

FIgURE 11: Correlation between $\beta_{1}$ and $\mu$ for $A_{1}=0.01, A_{2}=0.02$, and $\beta_{2}=0.02,0.04,0.06,0.08$.

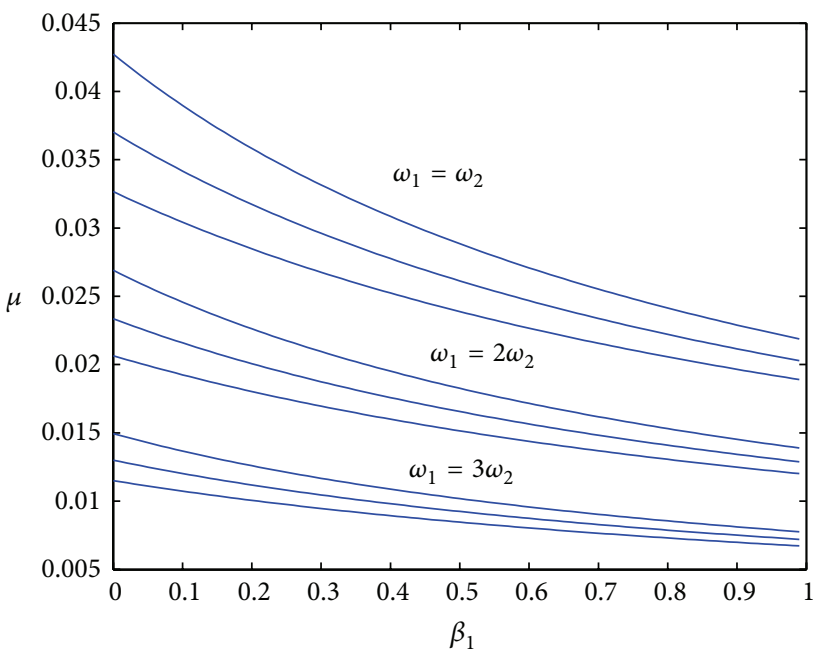

Figure 12: Combined figure between $\mu$ and $\beta_{1}\left(\beta_{2}=0.01, A_{1}=\right.$ 0.01 , and $A_{2}=0.01,0.02,0.03$ ).

theorem, linear canonical transformation of variations as given by Manju and Choudhry [1] is used which is given as

$$
\left(q_{1}, q_{2}, p_{1}, p_{2}\right)=\left(q_{1}^{\prime}, q_{2}^{\prime}, p_{1}^{\prime}, p_{2}^{\prime}\right) N
$$

where

$$
N=\left[\begin{array}{cccc}
a_{1} & a_{1} c_{1} & -a_{1} c_{1} & a_{1}\left(1-\omega_{1}^{2} b_{1}\right) \\
a_{2} & a_{2} c_{2} & -a_{2} c_{2} & a_{2}\left(1-\omega_{2}^{2} b_{2}\right) \\
0 & a_{1} b_{1} & a_{1}\left(1-b_{1}\right) & a_{1} c_{1} \\
0 & -a_{2} b_{2} & -a_{2}\left(1-b_{2}\right) & -a_{2} c_{2}
\end{array}\right],
$$


where

$$
\begin{aligned}
& a_{1}=\frac{1}{2}\left(\frac{2 l_{1}}{\omega_{1}^{2}-1 / 2}\right)^{1 / 2} ; \\
& a_{2}=\frac{1}{2}\left(\frac{2 l_{2}}{\omega_{2}^{2}-1 / 2}\right)^{1 / 2}, \\
& l_{1}=1+\omega_{1}^{2}+2 H_{02} ; \\
& l_{2}=1+\omega_{2}^{2}+2 H_{02} \\
& b_{1}=\frac{2}{l_{1}} ; \\
& b_{2}=\frac{2}{l_{2}} \\
& c_{1}=\frac{-H_{11}}{l_{1}} ; \\
& c_{2}=\frac{-H_{11}}{l_{2}} .
\end{aligned}
$$

The transformation (31) reduces the Hamiltonian to the following form:

$$
\begin{aligned}
H= & \frac{1}{2}\left(p_{1}^{\prime 2}+\omega_{1}^{2} q_{1}^{\prime 2}\right)-\frac{1}{2}\left(p_{2}^{\prime 2}+\omega_{2}^{2} q_{2}^{\prime 2}\right) \\
& +\sum_{\alpha+\gamma=3}^{\infty} h_{\alpha_{1} \alpha_{2} \gamma_{1} \gamma_{2}} q_{1}^{\prime \alpha_{1}} q_{2}^{\prime \alpha_{2}} p_{1}^{\prime \gamma_{1}} p_{2}^{\prime \gamma_{2}},
\end{aligned}
$$

where $\alpha=\alpha_{1}+\alpha_{2} ; \gamma=\gamma_{1}+\gamma_{2}$.

The Hamiltonian $\mathrm{H}_{3}$ can be expanded in the following form:

$$
\begin{aligned}
H_{3}= & H_{0003} p_{2}^{3}+H_{0030} p_{1}^{3}+H_{0300} q_{2}^{3}+H_{3000} q_{1}^{3} \\
& +H_{2100} q_{1}^{2} q_{2}+H_{2010} q_{1}^{2} p_{1}+\cdots+H_{1011} q_{1} p_{1} p_{2} .
\end{aligned}
$$

Similarly $\mathrm{H}_{4}$ can be expanded as

$$
\begin{aligned}
H_{4}= & H_{0004} p_{2}^{4}+H_{0040} p_{1}^{4}+H_{0400} q_{2}^{4}+H_{4000} q_{1}^{4}+\cdots \\
& +H_{1111} q_{1} q_{2} p_{1} p_{2} .
\end{aligned}
$$

The coefficient of third and fourth order terms of $h_{\alpha_{1} \alpha_{2} \gamma_{1} \gamma_{2}}^{\prime}$ and $h_{\alpha_{1} \alpha_{2} \gamma_{1} \gamma_{2}}$ given by Kumar and Choudhry [2] can be listed as

$$
\begin{aligned}
& h_{0030}=a_{1}^{3} b_{1}^{3} H_{0300} ; \\
& h_{3000}=a_{1}^{3}\left(H_{3000}+c_{1} H_{2100}+c_{1}^{2} H_{2100}+c_{1}^{3} H_{0300}\right) ;
\end{aligned}
$$

$$
\begin{aligned}
& h_{1020}=a_{1}^{3} b_{1}^{2}\left(H_{1200}+3 c_{1} H_{0300}\right) ; \\
& h_{2010}=a_{1}^{3} b_{1}\left(H_{2100}+2 c_{1} H_{1200}+3 c_{1}^{2} H_{0300}\right) \text {; } \\
& h_{0120}=a_{1}^{2} a_{2} b_{1}^{2}\left(H_{1200}+3 c_{2} H_{0300}\right) \text {; } \\
& h_{2001}=-a_{1}^{2} a_{2} b_{2}\left(H_{2100}+2 c_{1} H_{1200}+3 c_{1}^{2} H_{0300}\right) \text {; } \\
& h_{1011}=-2 a_{1}^{2} a_{2} b_{1} b_{2}\left(H_{1200}+3 c_{1} H_{0300}\right) \text {; } \\
& h_{1110}=2 a_{1}^{2} a_{2} b_{1}\left[H_{2100}+\left(c_{1}+c_{2}\right) H_{1200}+3 c_{1} c_{2} H_{0300}\right] \text {; } \\
& h_{0021}=-3 a_{1}^{2} b_{1}^{2} b_{2} H_{0300} \text {; } \\
& h_{2100}=a_{1}^{2} a_{2}\left[3 H_{3000}+\left(2 c_{1}+c_{2}\right) H_{2100}\right. \\
& \left.+c_{1}\left(c_{1}+2 c_{2}\right) H_{1200}+3 c_{1}^{2} c_{2} H_{0300}\right] ; \\
& h_{1002}=a_{1} b_{1}^{2} a_{2}^{2}\left(H_{1200}+3 c_{1} H_{0300}\right) \text {; } \\
& h_{0210}=a_{1} a_{2}^{2} b_{1}\left(H_{2100}+2 c_{2} H_{1200}+3 c_{2}^{2} H_{0300}\right) \text {; } \\
& h_{0012}=3 a_{1} a_{2}^{2} b_{2}^{2} b_{1} H_{0300} \text {; } \\
& h_{1200}=a_{2}^{2} a_{1}\left[3 H_{3000}+\left(c_{1}+2 c_{2}\right) H_{2100}\right. \\
& \left.+c_{2}\left(2 c_{1}+c_{2}\right) H_{1200}+3 c_{1} c_{2}^{2} H_{0300}\right] ; \\
& h_{0111}=-2 a_{1}^{2} a_{2}^{2} b_{1} b_{2}\left(H_{1200}+3 c_{2} H_{0300}\right) \text {; } \\
& h_{1101}=-2 a_{2}^{2} a_{1} b_{2}\left[H_{2100}+\left(c_{1}+c_{2}\right) H_{1200}\right. \\
& \left.+3 c_{1} c_{2} H_{0300}\right] \text {; } \\
& h_{0102}=a_{2}^{3} b_{21}^{2}\left(H_{1200}+3 c_{2} H_{0300}\right) \text {; } \\
& h_{0201}=-a_{2}^{3} b_{2}\left(H_{2100}+2 c_{2} H_{1200}+3 c_{2}^{2} H_{0300}\right) \text {; } \\
& h_{0102}=a_{2}^{3} b_{2}^{2}\left(H_{1200}+3 c_{2} H_{0300}\right) \text {; } \\
& h_{0003}=-a_{2}^{3} b_{2}^{3} H_{0300} \text {; } \\
& h_{0300}=a_{2}^{3}\left(H_{3000}+c_{2} H_{2100}+c_{2}^{2} H_{1200}+c_{2}^{3} H_{0300}\right) \text {; } \\
& h_{0040}=a_{1}^{4} b_{1}^{4} H_{0400} \text {; } \\
& h_{4000}=a_{1}^{4}\left(H_{4000}+c_{1} H_{3100}+c_{1}^{2} H_{2200}+c_{1}^{3} H_{1300}\right. \\
& \left.+c_{1}^{4} H_{0400}\right) \text {; } \\
& h_{2020}=a_{1}^{4} b_{1}^{2}\left(H_{2200}+3 c_{1} H_{1300}+6 c_{1}^{2} H_{0400}\right) \text {; } \\
& h_{0022}=6 a_{1}^{2} a_{2}^{2} b_{1}^{2} b_{2}^{2} H_{0400} \text {; } \\
& h_{2200}=a_{1}^{2} a_{2}^{2}\left[6 H_{4000}+3\left(c_{1}+c_{2}\right) H_{3100}\right. \\
& +\left(c_{1}^{2}+4 c_{1} c_{2}+c_{2}^{2}\right) H_{2200}+3 c_{1} c_{2}\left(c_{1}+c_{2}\right) H_{1300} \\
& \left.+6 c_{1}^{2} c_{2}^{2} H_{0400}\right] \text {; } \\
& h_{2002}=a_{1}^{2} a_{2}^{2} b_{2}^{2}\left(H_{2200}+3 c_{1} H_{1300}+6 c_{1}^{2} H_{0400}\right) \text {; }
\end{aligned}
$$




$$
\begin{aligned}
& h_{0220}=a_{1}^{2} a_{2}^{2} b_{1}^{2}\left(H_{2200}+3 c_{2} H_{1300}+6 c_{2}^{2} H_{0400}\right) \text {; } \\
& h_{0004}=a_{2}^{4} b_{2}^{4} H_{0400} \text {; } \\
& h_{0400}=a_{2}^{4}\left(H_{4000}+c_{2} H_{3100}+c_{2}^{2} H_{2200}+c_{2}^{3} H_{1300}\right. \\
& \left.+c_{2}^{4} H_{0400}\right) \text {; } \\
& h_{0202}=a_{2}^{4} b_{2}^{2}\left(H_{2200}+3 c_{2} H_{1300}+6 c_{2}^{2} H_{0400}\right) \text {; } \\
& h_{0013}=-4 a_{1} a_{2}^{3} b_{1} b_{2}^{3} H_{0400} \text {; } \\
& h_{1300}=a_{1} a_{2}^{3}\left[4 H_{4000}+\left(c_{1}+3 c_{2}\right) H_{3100}+4 c_{1} c_{2}^{3} H_{0400}\right. \\
& \left.+2 c_{2}\left(c_{1}+c_{2}\right) H_{2200}+c_{2}^{2}\left(3 c_{1}+c_{2}\right) H_{1300}\right] ; \\
& h_{1102}=a_{1} a_{2}^{3} b_{2}^{2}\left[2 H_{2200}+3\left(c_{1}+c_{2}\right) H_{1300}\right. \\
& \left.+12 c_{1} c_{2} H_{0400}\right] \text {; } \\
& h_{0211}=-2 a_{2}^{3} a_{1} b_{1} b_{2}\left[H_{2200}+3 c_{2} H_{1300}+6 c_{2}^{2} H_{0400}\right] \text {; } \\
& h_{0112}=3 a_{1} a_{2}^{3} b_{1} b_{2}^{2}\left(H_{1300}+4 c_{1} H_{0400}\right) \text {; } \\
& h_{1003}=-a_{1} a_{2}^{3} b_{2}^{3}\left(H_{1300}+4 c_{1} H_{0400}\right) \text {; } \\
& h_{1201}=-a_{1} a_{2}^{3} b_{2}\left[3 H_{3100}+2\left(c_{1}+2 c_{2}\right) H_{2200}\right. \\
& \left.+3 c_{2}\left(2 c_{1}+c_{2}\right) H_{1300}+12 c_{1} c_{2}^{2} H_{0400}\right] \text {; } \\
& h_{0310}=a_{1} a_{2}^{3} b_{1}\left(H_{3100}+2 c_{2} H_{2200}+3 c_{2}^{2} H_{1300}\right. \\
& \left.+4 c_{2}^{2} H_{0400}\right) \text {. }
\end{aligned}
$$

Again the Hamiltonian is reduced to a more convenient form which is suitable for further investigation by using the following canonical transformation:

$$
\begin{aligned}
& q_{1}^{\prime}=\frac{1}{2} q_{1}^{\prime \prime}+\frac{i}{\omega_{1}} p_{1}^{\prime \prime} ; \\
& p_{1}^{\prime}=\frac{1}{2} i \omega_{1} q_{1}^{\prime \prime}+p_{1}^{\prime \prime} ; \\
& q_{2}^{\prime}=-\frac{1}{2} q_{2}^{\prime \prime}+\frac{1}{\omega_{2}} p_{2}^{\prime \prime} ; \\
& p_{2}^{\prime}=-\frac{1}{2} i \omega q_{2}^{\prime \prime}+i p_{2}^{\prime \prime} .
\end{aligned}
$$

Thus, the Hamiltonian (34) may be written as

$$
\begin{aligned}
H= & i \omega_{1} q_{1}^{\prime \prime} p_{1}^{\prime \prime}+i \omega_{2} q_{2}^{\prime \prime} p_{2}^{\prime \prime} \\
& +\sum_{\alpha+\gamma=3}^{\infty} h_{\alpha_{1} \alpha_{2} \gamma_{1} \gamma_{2}}^{\prime} q_{1}^{\prime \prime \alpha_{1}} q_{2}^{\prime \prime \alpha_{2}} p_{1}^{\prime \prime \gamma_{1}} p_{2}^{\prime \prime \gamma_{2}}
\end{aligned}
$$

As explained by Kumar and Choudhry [2], consider the following.
If $h_{\alpha_{1} \alpha_{2} \gamma_{1} \gamma_{2}}^{\prime}=x_{\alpha_{1} \alpha_{2} \gamma_{1} \gamma_{2}}+i y_{\alpha_{1} \alpha_{2} \gamma_{1} \gamma_{2}}$ then

$$
\begin{aligned}
& x_{0030}=h_{0030}-\frac{1}{\omega_{1}^{2}} h_{2010} \text {; } \\
& y_{0030}=\frac{1}{\omega_{1}} h_{1020}-\frac{1}{\omega_{1}^{3}} h_{3000} \\
& x_{1020}=-\frac{1}{2} h_{1020}-\frac{3}{2 \omega_{1}^{2}} h_{3000} \text {; } \\
& y_{1020}=\frac{3 \omega_{1}}{2} h_{0030}+\frac{1}{2 \omega_{1}} h_{2010} \text {; } \\
& x_{0120}=-\frac{\omega_{2}}{2} h_{0021}+\frac{1}{2 \omega_{1}} h_{1110}+\frac{\omega_{2}}{2 \omega_{1}} h_{2001} \text {; } \\
& y_{0120}=-\frac{1}{2} h_{0120}-\frac{\omega_{2}}{2 \omega_{1}} h_{1011}+\frac{1}{2 \omega_{1}^{2}} h_{2100} \text {; } \\
& x_{1011}=-\omega_{1} h_{0021}-\frac{1}{\omega_{1}} h_{2001} ; \\
& y_{0021}=h_{0021}+\frac{1}{\omega_{1} \omega_{2}} h_{1110}-\frac{1}{\omega_{1}^{2}} h_{2001} \text {; } \\
& x_{1002}=-\frac{\omega_{1}}{2 \omega_{2}} h_{0111}-\frac{1}{2} h_{1002}+\frac{1}{2 \omega_{2}^{2}} h_{1200} \text {; } \\
& y_{1002}=-\frac{\omega_{1}}{2} h_{0012}+\frac{\omega_{1}}{2 \omega_{1}^{2}} h_{0210}+\frac{1}{2 \omega_{2}^{2}} h_{1101} \text {; } \\
& x_{0012}=-h_{0012}+\frac{1}{\omega_{2}^{2}} h_{0210}-\frac{1}{\omega_{1} \omega_{2}} h_{1101} \text {; } \\
& y_{0012}=\frac{1}{\omega_{2}} h_{0111}-\frac{1}{\omega_{1}} h_{1002}+\frac{1}{\omega_{1} \omega_{2}^{2}} h_{1200} \text {; } \\
& x_{0111}=\frac{\omega_{2}}{\omega_{1}} h_{1002}+\frac{1}{\omega_{1} \omega_{2}} h_{1200} \text {; } \\
& y_{0111}=-\omega_{2} h_{0012}-\frac{1}{\omega_{2}} h_{0210} ; \\
& x_{0201}=\frac{-\omega_{2}}{4} h_{0102}-\frac{3}{4 \omega_{2}} h_{0300} ; \\
& y_{0201}=\frac{-3 \omega_{2}^{2}}{4} h_{0003}+\frac{1}{4} h_{0201} \text {; } \\
& x_{0003}=\frac{-1}{\omega_{2}} h_{0102}+\frac{1}{\omega_{2}^{3}} h_{0300} \text {; } \\
& y_{0003}=-h_{0003}+\frac{1}{\omega_{2}^{2}} h_{0201} \text {. }
\end{aligned}
$$

The other ten coefficients $h_{\alpha_{1} \alpha_{2} \gamma_{1} \gamma_{2}}^{\prime}$ of third order terms of (39) are obtained by the formula

$$
\begin{aligned}
& h_{\alpha_{1} \alpha_{2} \gamma_{1} \gamma_{2}}^{\prime} \\
& \quad=\left(y_{\alpha_{1} \alpha_{2} \gamma_{1} \gamma_{2}}+i x_{\alpha_{1} \alpha_{2} \gamma_{1} \gamma_{2}}\right)\left(\frac{-\omega_{1}}{2}\right)^{\gamma_{1}-\alpha_{1}}\left(\frac{-\omega_{2}}{2}\right)^{\gamma_{2}-\alpha_{2}} .
\end{aligned}
$$


If we denote $\left(\alpha_{1}-\gamma_{1}\right)+\left(\alpha_{2}-\gamma_{2}\right)=a_{\alpha \gamma}$ and consider $\mid \alpha_{1}-$ $\gamma_{1}|+| \alpha_{2}-\gamma_{2} \mid=3$ and if it is found that $a_{\alpha \gamma} \neq$ an integer, then it implies that resonance of the third order is absent (Kumar and Choudhry [14]).

Using Birkhoff's transformation $\left(q_{j}^{\prime \prime}, p_{j}^{\prime \prime}\right) \rightarrow\left(q_{j}^{\prime \prime \prime}, p_{j}^{\prime \prime \prime}\right)$, all the third order terms from the Hamiltonian (39) are nullified provided third order resonance does not occur. This transformation is introduced by means of the generating function which is given as follows:

$$
s=q_{1}^{\prime \prime} p_{1}^{\prime \prime \prime}+q_{2}^{\prime \prime \prime} p_{2}^{\prime \prime \prime}+s_{3}+s_{4}
$$

where

$$
\begin{aligned}
& q_{i}^{\prime \prime \prime}=q_{i}^{\prime \prime}+\frac{\partial s_{3}}{\partial p_{i}^{\prime \prime \prime}}+\frac{\partial s_{4}}{\partial p_{i}^{\prime \prime \prime}} ; \\
& p_{i}^{\prime \prime}=p_{i}^{\prime \prime \prime}+\frac{\partial s_{3}}{\partial q_{i}^{\prime \prime}}+\frac{\partial s_{4}}{\partial q_{i}^{\prime \prime}} ; \quad(i=1,2) .
\end{aligned}
$$

From (39) and (43), expanding and equating the terms of the same degree on the two sides, we obtain

$$
\begin{aligned}
& H_{2}^{\prime}\left(q_{1}^{\prime \prime}, q_{2}^{\prime \prime}, p_{1}^{\prime \prime \prime}, p_{2}^{\prime \prime \prime}\right)=H_{2}\left(q_{1}^{\prime \prime}, q_{2}^{\prime \prime}, p_{1}^{\prime \prime \prime}, p_{2}^{\prime \prime \prime}\right) ; \\
& H_{3}^{\prime}\left(q_{1}^{\prime \prime}, q_{2}^{\prime \prime}, p_{1}^{\prime \prime \prime}, p_{2}^{\prime \prime \prime}\right) \\
& =\sum_{i=1}^{2}\left[-\frac{\partial s_{3}}{\partial p_{i}^{\prime \prime \prime}} \frac{\partial H_{2}^{\prime}}{\partial p_{i}^{\prime \prime \prime}}+\frac{\partial s_{3}}{\partial q_{i}^{\prime \prime}} \frac{\partial H_{2}}{\partial p_{i}^{\prime \prime \prime}}\right] \\
& \quad+H_{3}\left(q_{1}^{\prime \prime}, q_{2}^{\prime \prime}, p_{1}^{\prime \prime \prime}, p_{2}^{\prime \prime \prime}\right) ; \\
& \sum_{i=1}^{2}\left[-\frac{\partial s_{4}}{\partial p_{i}^{\prime \prime \prime}} \frac{\partial H_{2}^{\prime}}{\partial q_{i}^{\prime \prime \prime}}+\frac{\partial s_{4}}{\partial q_{i}^{\prime \prime}} \frac{\partial H_{2}}{\partial p_{i}^{\prime \prime \prime}}\right]+K_{4}=0 ; \\
& H_{4}^{\prime}=\sum_{i=1}^{2}\left[-\frac{\partial s_{3}}{\partial p_{i}^{\prime \prime \prime}} \frac{\partial H_{3}^{\prime}}{\partial q_{i}^{\prime \prime}}+\frac{\partial s_{3}}{\partial q_{i}^{\prime \prime}} \frac{\partial H_{3}}{\partial p_{i}^{\prime \prime \prime}}\right]+H_{4}-K_{4},
\end{aligned}
$$

where $K_{4}$ is the term other than the homogeneous ones in $q_{1} p_{1}$ and $q_{2} p_{2}$. In (44) the new variables $q_{1}^{\prime \prime \prime}$ and $p_{2}^{\prime \prime \prime}$ can be replaced by $q_{1}^{\prime \prime}$ and $p_{2}^{\prime \prime}$ on both sides of (44) by implicit function theorem.

Since our system is autonomous $\partial s_{3} / \partial t=\partial s_{4} / \partial t=0$.

If we put

$$
\begin{aligned}
& H_{3}=\sum_{\alpha+\gamma=3}^{\infty} h_{\alpha_{1} \alpha_{2} \gamma_{1} \gamma_{2}} q_{1}^{\prime \prime \prime \alpha_{1}} q_{2}^{\prime \prime \prime \alpha_{2}} p_{1}^{\prime \prime \prime \gamma_{1}} p_{2}^{\prime \prime \prime} \gamma_{2}, \\
& s_{3}=\sum_{\alpha+\gamma=3}^{\infty} g_{\alpha_{1} \alpha_{2} \gamma_{1} \gamma_{2}} q_{1}^{\prime \prime \prime \alpha_{1}} q_{2}^{\prime \prime \prime \alpha_{2}} p_{1}^{\prime \prime \prime \gamma_{1}} p_{2}^{\prime \prime \prime \gamma_{2}},
\end{aligned}
$$

using (44), we get

$$
g_{\alpha_{1} \alpha_{2} \gamma_{1} \gamma_{2}}=\frac{i h_{\alpha_{1} \alpha_{2} \gamma_{1} \gamma_{2}}^{\prime}}{\left(\alpha_{1}-\gamma_{1}\right) \omega_{1}+\left(\alpha_{2}-\gamma_{2}\right) \omega_{2}} .
$$

With the help of third equation of (44), the new Hamiltonian, inclusive of the fourth order terms, is given as

$$
\begin{aligned}
H^{\prime}= & i \omega_{1} q_{1}^{\prime \prime \prime} p_{1}^{\prime \prime \prime}+i \omega_{2} q_{2}^{\prime \prime \prime} p_{2}^{\prime \prime \prime}-c_{20}\left(q_{1}^{\prime \prime \prime} p_{1}^{\prime \prime \prime}\right)^{2} \\
& +c_{11}\left(q_{1}^{\prime \prime \prime} p_{1}^{\prime \prime \prime}\right)\left(q_{2}^{\prime \prime \prime} p_{2}^{\prime \prime \prime}\right)-c_{02}\left(q_{2}^{\prime \prime \prime} p_{2}^{\prime \prime \prime}\right)^{2}+\cdots,
\end{aligned}
$$

where

$$
K_{4}=H_{4}\left(q_{1}^{\prime \prime \prime}, q_{2}^{\prime \prime \prime}, p_{1}^{\prime \prime \prime}, p_{2}^{\prime \prime \prime}\right)-h_{2020}^{\prime} q_{1}^{\prime \prime \prime 2} p_{1}^{\prime \prime \prime 2}
$$$$
-h_{1111}^{\prime}\left(q_{1}^{\prime \prime \prime} p_{1}^{\prime \prime \prime}\right)\left(q_{2}^{\prime \prime \prime} p_{2}^{\prime \prime \prime}\right)
$$$$
-h_{0202}^{\prime} q_{2}^{\prime \prime \prime 2} p_{2}^{\prime \prime \prime 2}
$$

$$
c_{20}=-h_{2020}^{\prime}-\frac{3 \omega_{1}^{2}}{8}\left(x_{0030}^{2}+y_{0030}^{2}\right)
$$$$
-\frac{3}{2}\left(x_{1020}^{2}+y_{1020}^{2}\right)+\frac{1}{2}\left(x_{1011}^{2}+y_{1011}^{2}\right)
$$$$
-\frac{\omega_{1}^{2}}{2 \omega_{2}\left(2 \omega_{1}-\omega_{2}\right)}\left(x_{0120}^{2}+y_{0120}^{2}\right)
$$$$
+\frac{\omega_{1}^{2} \omega_{2}}{8\left(2 \omega_{1}+\omega_{2}\right)} \frac{3}{2}\left(x_{0021}^{2}+y_{0021}^{2}\right) \text {, }
$$

$$
c_{11}=-h_{1111}^{\prime}-\frac{2 \omega_{2}^{2}}{\omega_{1}\left(\omega_{1}-2 \omega_{2}\right)}\left(x_{1002}^{2}+y_{1002}^{2}\right)
$$

$$
+\frac{\omega_{1} \omega_{2}^{2}}{2\left(\omega_{1}+2 \omega_{2}\right)}\left(x_{0012}^{2}+y_{0012}^{2}\right)
$$$$
-\frac{\omega_{2} \omega_{1}^{2}}{2\left(2 \omega_{1}+\omega_{2}\right)}\left(x_{0021}^{2}+y_{0021}^{2}\right)
$$$$
-\frac{2 \omega_{1}^{2}}{\left(2 \omega_{1}-\omega_{2}\right)}\left(x_{0120}^{2}+y_{0120}^{2}\right)
$$$$
+2\left(x_{0111} x_{1020}+y_{0111} y_{1020}\right)
$$$$
-\frac{4}{\omega_{2}}\left(x_{0201} y_{1011}+x_{1011} y_{0201}\right) \text {; }
$$

$$
\begin{aligned}
c_{02}= & h_{0202}^{\prime}+\frac{3 \omega_{2}^{2}}{8}\left(x_{0003}^{2}+y_{0003}^{2}\right) \\
& +\frac{6}{\omega_{2}^{2}}\left(x_{0201}^{2}+y_{0201}^{2}\right) \\
& -\frac{\omega_{2}^{2}}{2 \omega_{1}\left(\omega_{1}-2 \omega_{2}\right)}\left(x_{1002}^{2}+y_{1002}^{2}\right) \\
& -\frac{1}{2}\left(x_{0111}^{2}+y_{0111}^{2}\right) \\
& -\frac{\omega_{1} \omega_{2}^{2}}{8\left(\omega_{1}+2 \omega_{2}\right)}\left(x_{0012}^{2}+y_{0012}^{2}\right) ;
\end{aligned}
$$




$$
\begin{aligned}
& h_{2020}^{\prime}=-\frac{3}{2} \omega_{1}^{2} h_{0040}-\frac{3}{2 \omega_{1}^{2}} h_{4000}-\frac{1}{2} h_{2020} ; \\
& h_{1111}^{\prime}=\omega_{1} \omega_{2} h_{0022}+\frac{1}{\omega_{1} \omega_{2}} h_{2200}+\frac{\omega_{1}}{\omega_{2}} h_{0220}+\frac{\omega_{2}}{\omega_{1}} h_{2002} ; \\
& h_{0202}^{\prime}=\frac{-3}{2} \omega_{2}^{2} h_{0004}-\frac{3}{2 \omega_{2}^{2}} h_{0400}-\frac{1}{2} h_{0202} ;
\end{aligned}
$$

now, KAM theorem is applied which is best suited for the problem which is being defined and is stated as follows.

If the Hamiltonian of the perturbed motion satisfy the following given conditions mentioned below as:

(1) The characteristics equation of the system with $\mathrm{H}_{2}$ has pure imaginary roots and the frequencies $\omega_{1}, \omega_{2}$ satisfy the inequality

$$
\begin{aligned}
& n_{1} \omega_{1}+n_{2} \omega_{2} \neq 0 \\
& \text { when }\left|n_{1}\right|+\left|n_{2}\right| \leq 4 \quad\left(n_{j} \text { are integers }\right) .
\end{aligned}
$$

(2) Consider $D=c_{20} \omega_{2}^{2}+c_{11} \omega_{1} \omega_{2}+c_{02} \omega_{1}^{2} \neq 0$, as given by Kumar and Choudhry [2].

If all the above mentioned conditions are satisfied, then the equilibrium points are stable.

The value of $D$ is calculated with the help of the following formula:

$$
D=c_{20} \omega_{2}^{2}+c_{11} \omega_{1} \omega_{2}+c_{02} \omega_{1}^{2}
$$

where $c_{20}, c_{11}$, and $c_{02}$ are defined in (48).

Table 2 shows the values $D$ for different values of radiation pressures and oblateness parameters.

\section{Discussion and Conclusion}

The stability of the triangular equilibrium points in CR3BP is investigated when both primaries are radiating and oblate, under the nonresonance case using KAM theory. Recently Narayan and Singh [5] studied nonlinear stability considering both primaries as radiating in ER3BP and found that the binary systems are stable. It has been observed that in general the stability character remains the same even if oblateness factor is considered apart from radiation factor in circular cases. The following observation has been made assuming both primaries as radiating and oblateness:

(i) It is found that $\omega_{1}=\omega_{2}$ corresponds to the boundary region of the stability for the system, whereas the other two cases $\omega_{1}=2 \omega_{2}$ and $\omega_{1}=3 \omega_{2}$ correspond to the resonant cases. It has been observed that resonance of the third and the fourth order exists for all values of $\mu, \beta_{1}, \beta_{2}, A_{1}$, and $A_{2}$ taken.

(ii) It is noticed that for fixed values of radiation pressures and by varying oblateness parameters $\omega_{1}$ increases by increasing $\mu$ whereas $\omega_{2}$ decreases and becomes equal

\begin{tabular}{|c|c|c|c|c|c|}
\hline$\mu$ & $\beta_{1}$ & $\beta_{2}$ & $A_{1}$ & $A_{2}$ & Values of $D$ \\
\hline 0.001 & 0.87 & 0.29 & 0.01 & 0.02 & $-3.1202 \times 10^{9}$ \\
\hline 0.001 & 0.87 & 0.29 & 0.02 & 0.03 & $-4.8371 \times 10^{9}$ \\
\hline 0.001 & 0.87 & 0.29 & 0.04 & 0.05 & $-1.1754 \times 10^{7}$ \\
\hline 0.005 & 0.82 & 0.29 & 0.01 & 0.02 & $-2.7755 \times 10^{7}$ \\
\hline 0.005 & 0.82 & 0.29 & 0.02 & 0.03 & $-4.2670 \times 10^{7}$ \\
\hline 0.005 & 0.82 & 0.29 & 0.04 & 0.05 & $-6.5897 \times 10^{8}$ \\
\hline 0.010 & 0.72 & 0.29 & 0.01 & 0.02 & $-3.8443 \times 10^{6}$ \\
\hline 0.010 & 0.72 & 0.29 & 0.02 & 0.03 & $-5.8280 \times 10^{6}$ \\
\hline 0.010 & 0.72 & 0.29 & 0.04 & 0.05 & $-1.3814 \times 10^{7}$ \\
\hline 0.015 & 0.62 & 0.39 & 0.01 & 0.02 & $-1.5405 \times 10^{6}$ \\
\hline 0.015 & 0.62 & 0.39 & 0.02 & 0.03 & $-2.4736 \times 10^{6}$ \\
\hline 0.015 & 0.62 & 0.39 & 0.04 & 0.05 & $-6.9668 \times 10^{6}$ \\
\hline 0.025 & 0.52 & 0.49 & 0.01 & 0.02 & $-5.7810 \times 10^{5}$ \\
\hline 0.025 & 0.52 & 0.49 & 0.02 & 0.03 & $-1.0457 \times 10^{6}$ \\
\hline 0.025 & 0.52 & 0.49 & 0.04 & 0.05 & $-4.5781 \times 10^{6}$ \\
\hline 0.035 & 0.92 & 0.29 & 0.01 & 0.02 & $\begin{array}{l}-7.8501 \times 10^{4} \\
+1.1316 \times 10^{4} i\end{array}$ \\
\hline 0.035 & 0.92 & 0.29 & 0.02 & 0.03 & $\begin{array}{c}-1.4121 \times 10^{5} \\
+1.4800 \times \\
10^{4} i\end{array}$ \\
\hline 0.035 & 0.92 & 0.29 & 0.04 & 0.05 & $-9.9125 \times 10^{4}$ \\
\hline 0.038 & 0.72 & 0.49 & 0.01 & 0.02 & $6.8892 \times 10^{5}$ \\
\hline 0.038 & 0.72 & 0.49 & 0.02 & 0.03 & $-4.8483 \times 10^{6}$ \\
\hline 0.038 & 0.72 & 0.49 & 0.04 & 0.05 & $-2.3721 \times 10^{6}$ \\
\hline 0.038 & 0.62 & 0.39 & 0.01 & 0.02 & $6.9437 \times 10^{4}$ \\
\hline 0.038 & 0.62 & 0.39 & 0.02 & 0.03 & $3.2725 \times 10^{5}$ \\
\hline 0.038 & 0.62 & 0.39 & 0.04 & 0.05 & $-2.5116 \times 10^{6}$ \\
\hline 0.001 & 0.01 & 0.01 & 0.01 & 0.02 & $-3.2004 \times 10^{7}$ \\
\hline 0.001 & 0.01 & 0.01 & 0.02 & 0.03 & $-5.4067 \times 10^{7}$ \\
\hline 0.001 & 0.01 & 0.01 & 0.04 & 0.05 & $-1.5692 \times 10^{8}$ \\
\hline 0.005 & 0.01 & 0.02 & 0.01 & 0.02 & $-3.1222 \times 10^{5}$ \\
\hline 0.005 & 0.01 & 0.02 & 0.02 & 0.03 & $-5.2414 \times 10^{7}$ \\
\hline 0.005 & 0.01 & 0.02 & 0.04 & 0.05 & $-1.5044 \times 10^{6}$ \\
\hline 0.010 & 0.01 & 0.03 & 0.01 & 0.02 & $-3.0522 \times 10^{5}$ \\
\hline 0.010 & 0.01 & 0.03 & 0.02 & 0.03 & $-8.5874 \times 10^{4}$ \\
\hline 0.010 & 0.01 & 0.03 & 0.04 & 0.05 & $-2.3880 \times 10^{5}$ \\
\hline 0.015 & 0.01 & 0.04 & 0.01 & 0.02 & $-2.2203 \times 10^{4}$ \\
\hline 0.015 & 0.01 & 0.04 & 0.02 & 0.03 & $-3.5166 \times 10^{4}$ \\
\hline 0.015 & 0.01 & 0.04 & 0.04 & 0.05 & $-9.2124 \times 10^{4}$ \\
\hline 0.025 & 0.01 & 0.05 & 0.01 & 0.02 & $-4.5169 \times 10^{5}$ \\
\hline 0.025 & 0.01 & 0.05 & 0.02 & 0.03 & $-3.3881 \times 10^{4}$ \\
\hline 0.025 & 0.01 & 0.05 & 0.04 & 0.05 & $-3.2969 \times 10^{4}$ \\
\hline 0.030 & 0.01 & 0.06 & 0.01 & 0.02 & $3.4841 \times 10^{4}$ \\
\hline 0.030 & 0.01 & 0.06 & 0.02 & 0.03 & $1.7250 \times 10^{4}$ \\
\hline 0.030 & 0.01 & 0.06 & 0.04 & 0.05 & $-4.8836 \times 10^{4}$ \\
\hline 0.035 & 0.01 & 0.07 & 0.01 & 0.02 & 492.1240 \\
\hline 0.035 & 0.01 & 0.07 & 0.02 & 0.03 & $2.5112 \times 10^{4}$ \\
\hline 0.035 & 0.01 & 0.07 & 0.04 & 0.05 & $5.7581 \times 10^{5}$ \\
\hline 0.038 & 0.01 & 0.08 & 0.01 & 0.02 & 38.4651 \\
\hline 0.038 & 0.01 & 0.08 & 0.02 & 0.03 & $1.0408 \times 10^{3}$ \\
\hline 0.038 & 0.01 & 0.08 & 0.04 & 0.05 & $3.9251 \times 10^{4}$ \\
\hline
\end{tabular}
to the critical value (i.e., $\mu=\mu_{c}$ ).

(iii) From Table 2 it is clear that for $\mu=0.038, \beta_{1}=0.72$, $\beta_{2}=0.49, A_{1}=0.04, A_{2}=0.05$ and $\beta_{1}=0.62$,
TABLE 2: Values of $D$ at different values of eccentricity for different values of radiation pressure and oblateness parameter.

$\beta_{2}=0.39, A_{1}=0.01, A_{2}=0.02$, there is change in the sign of value of $D$. 
This change in sign suggests that for fixed $\mu=0.038$ and by slightly changing radiation pressure and oblateness parameters $D$ vanishes, where KAM theory is not applicable. It is also concluded that except at this point the equilibrium points are stable. Consider the following:

(i) It is clear from Table 2 that for $\mu=0.025, \beta_{1}=0.01$, $\beta_{2}=0.05, A_{1}=0.04, A_{2}=0.05$ and $\mu=0.030$, $\beta_{1}=0.01, \beta_{2}=0.06, A_{1}=0.01, A_{2}=0.02$ again there is change in sign of $D$. This suggests that for slight variation in radiation pressure and oblateness parameters and for $0.025 \leq \mu \leq 0.030$, the value of $D$ must be zero.

(ii) It is observed that for $\mu=0.030, \beta_{1}=0.01, \beta_{2}=0.06$, $A_{1}=0.02, A_{2}=0.03$ and $\mu=0.030, \beta_{1}=0.01$, $\beta_{2}=0.06, A_{1}=0.04, A_{2}=0.05 \mathrm{D}$ changes sign. This implies that when $\mu=0.030, \beta_{1}=0.01$, and $\beta_{2}=0.06$ and for slight variation in oblateness parameters, $D$ vanishes.

(iii) Also, it is found that when $\mu=0.038, \beta_{1}=0.62, \beta_{2}=$ $0.39, A_{1}=0.02, A_{2}=0.03$ and $\mu=0.038, \beta_{1}=0.01$, $\beta_{2}=0.06, A_{1}=0.04, A_{2}=0.05$, there is change in the sign of $D$.

From the above discussion the following conclusion can be drawn.

In all the above four cases considered, it is noticed that KAM theory fails. From the above discussion it is also clear that by varying one of the parameters and taking another parameter as fixed, there may be many cases where KAM theory fails and their motion can be found numerically which needs further investigation. It is observed that, except for some values of radiation pressure and oblateness parameters and for $\mu<0.0385209$ the triangular equilibrium points are stable.

\section{Conflict of Interests}

The authors declare that there is no conflict of interests regarding the publication of this paper.

\section{Acknowledgment}

The authors are grateful to the reviewers; theirs comments have been a great help in the improvement of the paper.

\section{References}

[1] Manju and R. K. Choudhry, "On the stability of triangular libration points taking into account the light pressure for the circular restricted problem of three bodies," Celestial Mechanics, vol. 36, no. 2, pp. 165-190, 1985.

[2] V. Kumar and R. K. Choudhry, "On the stability of the triangular libration points for the photogravitational circular restricted problem of three bodies when both of the attracting bodies are radiating as well," Celestial Mechanics, vol. 40, no. 2, pp. 155-170, 1987.

[3] K. B. Bhatnagar, U. Gupta, and R. Bhardwaj, "Effect of perturbed potentials on the non-linear stability of libration point $L_{4}$ in the restricted problem," Celestial Mechanics and Dynamical Astronomy, vol. 59, no. 4, pp. 345-374, 1994.

[4] V. V. Markellos, K. E. Papadakis, and E. A. Perdios, "Non-linear stability zones around triangular equilibria in the plane circular restricted three-body problem with oblateness," Astrophysics and Space Science, vol. 245, no. 1, pp. 157-164, 1996.

[5] A. Narayan and N. Singh, "Higher order resonance stability of triangular libration points for radiating primaries in ER3BP," International Journal of Advanced Astronomy, vol. 3, no. 2, pp. 26-39, 2015.

[6] J. M. A. Danby, "Stability of the triangular points in the elliptic restricted problem of three bodies," Astronomical Journal, vol. 69, pp. 165-172, 1964.

[7] A. Bennett, "Characteristic exponents of the five equilibrium solutions in the elliptically restricted problem," Icarus, vol. 4, no. 2, pp. 177-187, 1965.

[8] V. Szebebely, "Stability of the points of equilibrium in the restricted problem," The Astronomical Journal, vol. 72, pp. 7-9, 1967.

[9] A. P. Markeev, Libration Points in Celestial Mechanics and Cosmodynamics, Nauka, Moscow, Russia, 1978.

[10] D. Selaru and C. Cucu-Dumitrescu, "Infinitesimal orbit around Lagrange points in the elliptic restricted three body problem," Celestial Mechanics and Dynamical Astronomy, vol. 61, no. 4, pp. 333-346, 1995.

[11] P. P. Hallan and N. Rana, "The existence and stability of equilibrium points in the Robe's restricted three-body problem," Celestial Mechanics and Dynamical Astronomy, vol. 79, no. 2, pp. 145-155, 2001.

[12] J. Györgyey, "On the non-linear stability of motions around L5 in the elliptic restricted problem of the three bodies," Celestial Mechanics, vol. 36, no. 3, pp. 281-285, 1985.

[13] E. A. Grebenikov, "On the stability of the lagrangian triangular solutions of the restricted elliptic three body problem," Soviet Astronomy, vol. 8, no. 3, pp. 451-459, 1964.

[14] V. Kumar and R. K. Choudhry, "Nonlinear stability of the triangular libration points for the photo gravitational elliptic restricted problem of three bodies," Celestial Mechanics and Dynamical Astronomy, vol. 48, no. 4, pp. 299-317, 1990.

[15] V. V. Markellos, E. Perdios, and P. Labropoulou, "Linear stability of the triangular equilibrium points in the photogravitational elliptic restricted problem, I," Astrophysics and Space Science, vol. 194, no. 2, pp. 207-213, 1992.

[16] S. K. Sahoo and B. Ishwar, "Stability of collinear equilibrium points in the generalized photo gravitational elliptic restricted three-body problem," Bulletin of the Astronomical Society of India, vol. 28, pp. 579-586, 2000.

[17] G. E. Roberts, "Linear stability of the elliptic Lagrangian triangle solutions in the three-body problem," Journal of Differential Equations, vol. 182, no. 1, pp. 191-218, 2002.

[18] A. S. Zimovshchikov and V. N. Tkhai, "Instability of libration points and resonance phenomena in the photogravitational elliptic restricted three-body problem," Solar System Research, vol. 38, no. 2, pp. 155-163, 2004.

[19] M. K. Ammar, "The effect of solar radiation pressure on the Lagrangian points in the elliptic restricted three-body problem," Astrophysics and Space Science, vol. 313, no. 4, pp. 393-408, 2008.

[20] B. Érdi, E. Forgács-Dajka, I. Nagy, and R. Rajnai, "A parametric study of stability and resonance around $L_{4}$ in the elliptical restricted three body problem," Celestial Mechanics and Dynamical Astronomy, vol. 104, no. 1-2, pp. 145-158, 2009. 
[21] S. Kumar and B. Ishwar, "Location of collinear equilibrium points in the generalized photogravitational elliptical restricted three body problem," International Journal of Scientific \& Technology Research, vol. 3, no. 2, pp. 157-162, 2011.

[22] J. Singh and A. Umar, "Motion in the photogravitational elliptic restricted three-body problem under an oblate primary," Astronomical Journal, vol. 143, no. 5, article 109, 2012.

[23] J. Singh and A. Umar, "On the stability of triangular points in the elliptic R3BP under radiating and oblate primaries," Astrophysics and Space Science, vol. 341, no. 2, pp. 349-358, 2012.

[24] T. Usha, A. Narayan, and B. Ishwar, "Effects of radiation and triaxiality of primaries on triangular equilibrium points in elliptic restricted three body problem," Astrophysics \& Space Science, vol. 349, no. 1, pp. 151-164, 2014.

[25] A. Narayan and N. Singh, "Resonance stability of triangular equilibrium points in elliptical restricted three body problem under the radiating primaries," Astrophysics and Space Science, vol. 353, no. 2, pp. 441-455, 2014.

[26] A. Narayan and N. Singh, "Stability of triangular lagrangian points in elliptical restricted three body problem under the radiating binary systems," Astrophysics and Space Science, vol. 353, no. 2, pp. 457-464, 2014.

[27] A. Narayan and A. Shrivastava, "Existence of resonance stability of triangular equilibrium points in circular case of the planar elliptical restricted three-body problem under the oblate and radiating primaries around the binary system," Advances in Astronomy, vol. 2014, Article ID 287174, 17 pages, 2014.

[28] AM. Liapnuov, The general problem of stability of Motion [Ph.D. thesis], Academy of Science USSR, 1956.

[29] V. I. Arnold, "Proof of A. N. Kolmogorov's theorem on the preservation of quasiperiodic motions under small perturbations of the Hamiltonian," Russian Mathematical Surveys, vol. 18, no. 5, pp. 9-36, 1963.

[30] V. I. Arnold, "Small divisor problems in classical and celestial mechanics," Russian Mathematical Surveys, vol. 18, no. 6, pp. 85192, 1963. 

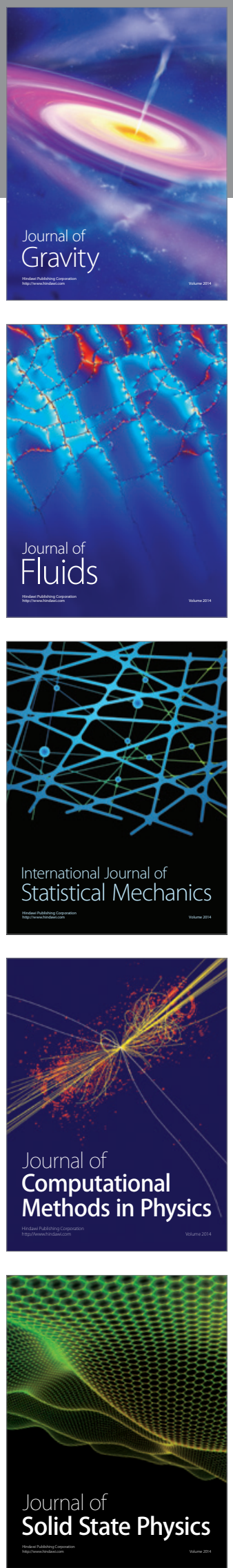

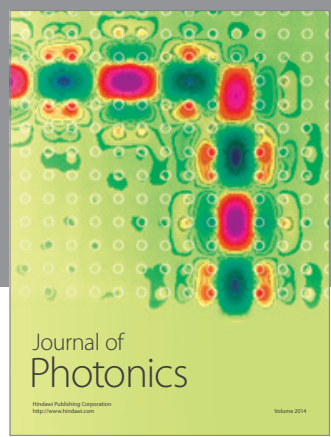

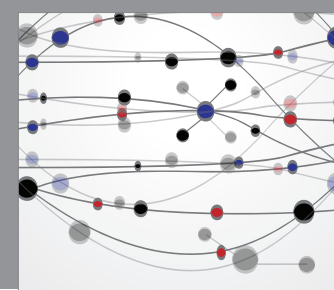

The Scientific World Journal

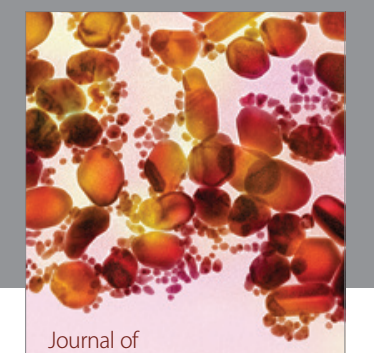

Soft Matter
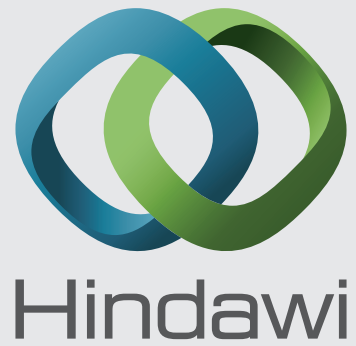

Submit your manuscripts at

http://www.hindawi.com
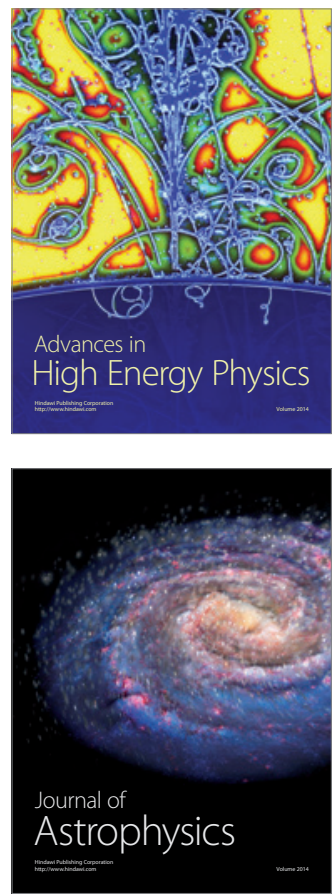
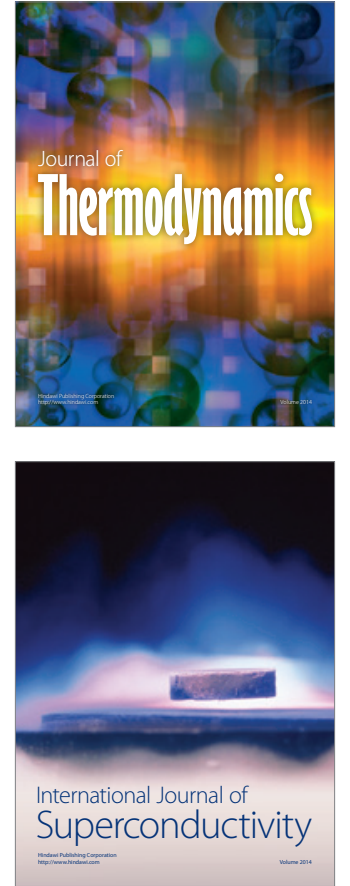
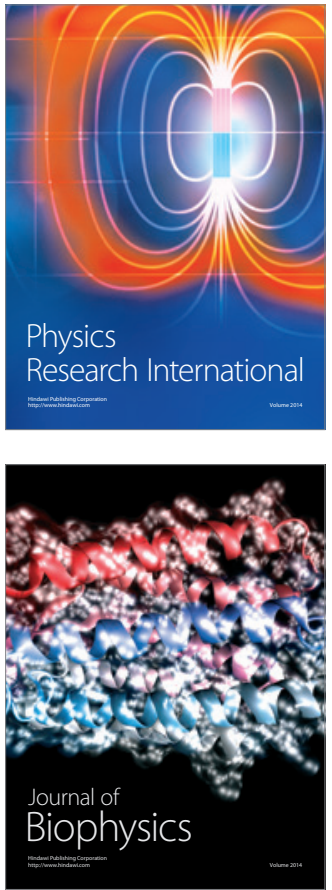
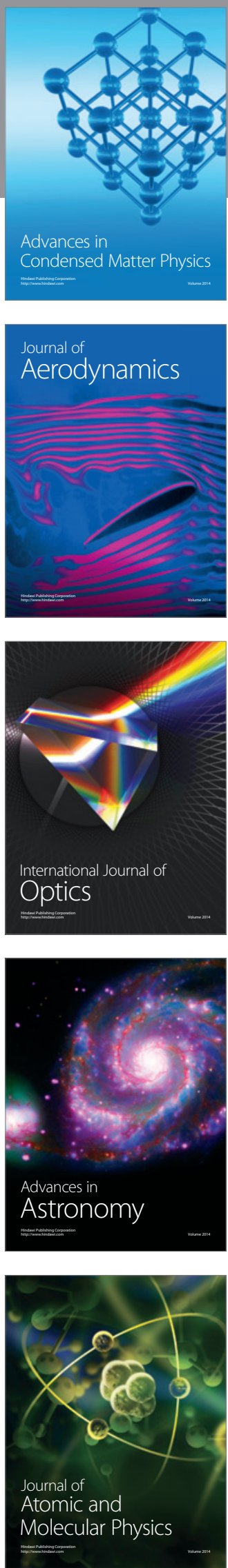